\title{
On Global Solutions of the Maxwell-Dirac Equations *
}

\author{
M. Flato ${ }^{1}$, Jacques Simon ${ }^{1}$, and Erik Taflin ${ }^{2}$ \\ 1 Département de Mathématiques, Université de Bourgogne, BP 138, \\ F-21004 Dijon Cedex, France \\ 2 The New Technology Division, Union des Assurances de Paris, 20 ter rue de Bezons, F-94411 \\ Courbevoie Cedex, France
}

\section{Dedicated to Walter Thirring on his $60^{\text {th }}$ birthday}

\begin{abstract}
We prove, for the Maxwell-Dirac equations in $1+3$ dimensions, that modified wave operators exist on a domain of small entire test functions of exponential type and that the Cauchy problem, in $R^{+} \times R^{3}$, has a unique solution for each initial condition (at $t=0$ ) which is in the image of the wave operator. The modification of the wave operator, which eliminates infrared divergences, is given by approximate solutions of the Hamilton-Jacobi equation, for a relativistic electron in an electromagnetic potential. The modified wave operator linearizes the Maxwell-Dirac equations to their linear part.
\end{abstract}

\section{Introduction}

Our basic understanding of electromagnetic interactions started with the fundamental discovery made by P. A. M. Dirac of the relativistic electron equation. After years of development it was only in the late forties that Schwinger, Feynman, and Tomonaga formulated Quantum Electro-Dynamics, the theory of fundamental interaction between electrons and photons. This theory, which starts with quantum operator-valued Maxwell-Dirac (M-D) coupled equations, ends up with a set of rules deduced non-mathematically from the previous, permitting to calculate with very high precision different electromagnetic processes. These are the famous Feynman rules. One can take the point of view that all the physics we need is contained in this set of rules, and therefore this is the only thing that matters. However it is quite tempting to believe that, though we did not deduce these rules mathematically, still the quantum $\mathrm{M}-\mathrm{D}$ equations must have some sense.

* This work is dedicated to Walter Thirring upon the occasion of his sixtieth birthday with appreciation and friendship 
Knowing in addition the fundamental role the Maxwell equations play in classical electromagnetism it is difficult not to believe that also in some sense the classical $\mathrm{M}-\mathrm{D}$ equation must have a fundamental importance.

The M-D equations being a system of coupled non-linear partial differential equations, different mathematical questions appear, of which the most fundamental are the following:

a) Existence and uniqueness of solutions for small times.

b) Existence and uniqueness of solutions for arbitrary times.

c) Dependence of the first two questions on the choice of type and size of initial data.

These questions have been debated in the literature at least in the last two decades, by many authors.

As early as 1966 Gross [8] obtained, to our knowledge for the first time, the existence and uniqueness of solutions, in suitable function spaces, for small times (local solutions). This interesting result has not permitted any extension to the problem of existence for arbitrary times. Kato [10] has later formalized this approach.

Several authors have considered the problem of existence for arbitrary times (global existence) of solutions for equations resembling the $\mathrm{M}-\mathrm{D}$ equations. Chadam found in 1972 [2] global solutions of the M-D equations in $1+1$ dimensions using the existence of constants of motion. He also proved [3] that for any fixed bounded region of space-time in $1+3$ dimensions there exists a solution of the $\mathrm{M}-\mathrm{D}$ equations in this region.

Using conformal invariance techniques Choquet-Bruhat [4] obtained global solutions for the zero mass Maxwell-Dirac-Klein-Gordon equations in the sector of global charge zero. This a priori general statement does not contain much relevance for the $\mathrm{M}-\mathrm{D}$ system because of the following reasons:

a) The electron has a strictly positive mass as is reflected in the M-D system and not zero mass.

b) The condition of zero total charge avoids definitely the M-D system as a subsystem of the "general" $\mathrm{M}-\mathrm{D}-\mathrm{K}-\mathrm{G}$ system.

This list of references to works on the $\mathrm{M}-\mathrm{D}$ equations is certainly not exhaustive. It will be remarked nevertheless that all works on the subject until now did not refer to the problem of existence of global solutions of the $1+3$ dimensional (over Minkowski space) realistic classical M-D systems. The aim of the present paper is to fill this gap.

More than 10 years ago we started the development of new techniques in the theory of covariant non-linear evolution equations [5-7]. In particular we developed cohomological techniques for existence and construction of linearization maps (transforming the non-linear equation into its linear part), which by definition are time independent. The existence of such maps insures in general (e.g. in the case in which the linear part is unitary) the existence of global solutions in time at least for small initial conditions, existence of non-linear superposition principles (like in the soliton case), and the existence of infinitely many constants of motion like in the case of integrable systems in 2 dimensions [1, 12, 13]. It can easily be understood that the usual Möller (or wave) operator in non-linear classical field theory is a linearization map, but certainly not every linearization map is a wave operator. 
From the Coulomb scattering problem in Schrödinger Quantum Mechanics we know that the modified Möller operators can be constructed and that they intertwine the interactive dynamics and the usual free dynamics. Taking this experience into account we have been able (essentially subtracting the classical infrared divergences) to construct a linearization map between the classical interactive $\mathrm{M}-\mathrm{D}$ equations and the usual linear part of these equations.

Our major new achievement in the present article is to establish the existence of global solutions (for $t \geqq 0$ ) of the $\mathrm{M}-\mathrm{D}$ system. Our result can and will be ameliorated in several ways:

a) Extension of globality to negative times.

b) Linearization of the whole Poincare action on the space of initial conditions.

c) Explicit study of interplay between gauge invariance and linearization techniques.

d) Introduction of more natural spaces of initial data such as spaces of $C^{\infty}$-vectors for the linear part, in view of studying large initial data.

It is our claim nevertheless that this article solves for the first time the problem of existence of global time solutions for the M-D systems.

The $\mathrm{M}-\mathrm{D}$ equations in $1+3$ dimensions reads in conventional notation (unitcharge $e=1, \gamma^{\mu} \gamma^{v}+\gamma^{v} \gamma^{\mu}=2 g^{\mu \nu}, g^{00}=1, g^{i i}=-1$ for $i=1,2,3$ and $g^{\mu v}=0$ for $\mu \neq v$ ):

$$
\begin{gathered}
\square A_{\mu}=\bar{\psi} \gamma_{\mu} \psi, \quad \mu=0,1,2,3, \\
\left(i \gamma^{\mu} \partial_{\mu}+m\right) \psi=A_{\mu} \gamma^{\mu} \psi, \quad m>0, \\
\partial_{\mu} A^{\mu}=0,
\end{gathered}
$$

where $\bar{\psi}=\psi^{+} \gamma^{0}, \psi^{+}$being the Hermitian conjugate of $\psi$. We write Eqs. (1.1a) and $(1.1 \mathrm{~b})$ as an evolution equation:

$$
\begin{aligned}
& \frac{d}{d t}\left(\begin{array}{c}
A_{\mu}(t) \\
\dot{A}_{\mu}(t)
\end{array}\right)=\left(\begin{array}{ll}
0 & I \\
\Delta & 0
\end{array}\right)\left(\begin{array}{l}
A_{\mu}(t) \\
A_{\mu}(t)
\end{array}\right)+\left(\begin{array}{c}
0 \\
\overline{\psi(t)} \gamma_{\mu} \psi(t)
\end{array}\right), \\
& \frac{d}{d t} \psi(t)=\mathscr{D} \psi(t)-i A_{\mu}(t) \gamma^{0} \gamma^{\mu} \psi(t),
\end{aligned}
$$

where $\mathscr{D}=-\sum_{j=1}^{3} \gamma^{0} \gamma^{j} \partial_{j}+i \gamma^{0} m, \partial_{j}=\partial / \partial x_{j}$.

The gauge condition (1.1c) takes on initial conditions $A\left(t_{0}\right), \dot{A}\left(t_{0}\right), \psi\left(t_{0}\right)$ at $t=t_{0}$ the form:

$$
\begin{gathered}
\dot{A}_{0}\left(t_{0}\right)+\sum_{i=1}^{3} \partial_{i} A^{i}\left(t_{0}\right)=0, \\
\Delta A_{0}\left(t_{0}\right)+\left|\psi\left(t_{0}\right)\right|^{2}+\sum_{i=1}^{3} \partial_{i} \dot{A}^{i}\left(t_{0}\right)=0 .
\end{gathered}
$$

It follows from Eqs. (1.2) and (1.3) that if $\psi\left(t_{0}\right) \neq 0$, then the electrical field $-\partial_{j} A_{0}\left(t_{0}\right)$ $+\dot{A}_{j}\left(t_{0}\right)$ does not decrease faster that $1 /|x|^{2}$ in space. The potential $A_{\mu}\left(t_{0}\right)$ will then in general not decrease faster than $1 /|x|$. The slow decrease in space of the potential and the conservation of the total charge make it necessary to introduce long range corrections in the definition of the wave operators. These corrections are 
introduced as follows. For a given Dirac field $\psi$, the electromagnetic potential $A_{\mu}(t)$ in Eq. (1.1a) is split into a long range part $G_{\mu}(t)$ defined by formula (3.14) and a short range part $A_{\mu}(t)-G_{\mu}(t)$ which is in $L^{2}\left(\mathbb{R}^{3}\right)$. Phase functions $S_{+}, S_{-}$, close to solutions $\mathbf{S}_{\varepsilon}$ of the classical Hamiltonian-Jacobi equation for a relativistic electron in an external field $G_{\mu}$, which is

$$
\left(\frac{\partial}{\partial t} \mathbf{S}_{\boldsymbol{\varepsilon}}(k, t)+G_{0}\left(-\nabla_{k} \mathbf{S}_{\boldsymbol{\varepsilon}}(k, t), t\right)\right)^{2}-\sum_{i=1}^{3}\left|k_{i}+G_{i}\left(-\nabla_{k} \mathbf{S}_{\boldsymbol{\varepsilon}}(k, t), t\right)\right|^{2}=m^{2}, \quad t \geqq 0
$$

are then determined by formula (3.40). The phase functions $S_{\varepsilon}$ serve to modify the dynamics $W_{0}$ (the evolution operator) of the outgoing electron states. $W_{0}(t, s)$ is the unitary evolution operator in $L^{2}\left(\mathbb{R}^{3}, C^{4}\right)$ defined by

$$
\left(W_{0}(t, s) f\right)^{\wedge}(k)=\sum_{\varepsilon= \pm} \exp \left(i S_{\varepsilon}(k, t)-i S_{\varepsilon}(k, s)\right) P_{\varepsilon}(k) \hat{f}(k), \quad t, s \geqq 0,
$$

where $P_{\varepsilon}(\partial), \varepsilon= \pm$ are the orthogonal projectors in $L^{2}\left(R^{3}, C^{4}\right)$ given by

$$
P_{\varepsilon}(k)=\frac{1}{2}\left(I+\varepsilon\left(-\sum_{j=1}^{3} \gamma^{0} \gamma^{j} k_{j}+m \gamma^{0}\right) \omega(k)^{-1}\right)
$$

and $\omega(k)=\left(m^{2}+|k|^{2}\right)^{1 / 2}$.

The Fourier transform is here defined by

$$
\hat{f}(k)=(2 \pi)^{-3 / 2} \int_{R^{3}} e^{-i k x} f(x) d x .
$$

The evolution operator $W_{0}$ satisfies

$$
\begin{gathered}
W_{0}(t, s) W_{0}(s, t)=I, \quad \frac{d}{d t} W_{0}(t, s)=\mathscr{L}(t) W_{0}(t, s), \\
\frac{d}{d t} W_{0}(t, s)=W_{0}(t, s) \mathscr{L}(s),
\end{gathered}
$$

where

$$
(\mathscr{L}(t) f)^{\wedge}(k)=i \sum_{\varepsilon= \pm} P_{\varepsilon}(k) \frac{\partial}{\partial t} S_{\varepsilon}(k, t) \hat{f}(k)
$$

Introduce

$$
\mu(t)=(-\Delta)^{-1 / 2} \sin \left((-\Delta)^{1 / 2} t\right), \quad \dot{\mu}(t)=\cos \left((-\Delta)^{1 / 2} t\right) .
$$

In order to solve the system (1.2) for given final states we integrate (1.2) into the form:

$$
\begin{gathered}
A_{\delta}(t)=\dot{\mu}(t) a_{\delta}+\mu(t) \dot{a}_{\delta}+\int_{\infty}^{t} \mu(t-s) \overline{\psi(s)} \gamma_{\delta} \psi(s) d s, \\
\psi(t)=W_{0}(t, 0) \alpha+\int_{\infty}^{t} W_{0}(t, s)\left(\mathscr{D}-i \gamma^{0} \gamma^{\mu} A_{\mu}(s)-\mathscr{L}(s)\right) \psi(s) d s, \quad t \geqq 0 .
\end{gathered}
$$

The correspondence $(a, \dot{a}, \alpha) \rightarrow(A, \psi)$ is not unique, because of the non-uniqueness of $\mathscr{L}$. This is not in contradiction with the fact that for given initial conditions $(A, \dot{A}, \psi)$ at $t=0$, the solution of the $\mathrm{M}-\mathrm{D}$ equations is unique. 
In this paper we will take $a_{\mu}, \dot{a}_{\mu}$ real and

$$
\hat{a}_{\mu}, \hat{\dot{a}}_{\mu} \in \mathscr{D}\left(R^{3}-\{0\}, C\right), \quad \hat{\alpha} \in \mathscr{D}\left(R^{3}, C^{4}\right),
$$

and we denote by $E_{+}$the space of such final states $(a, \dot{a}, \alpha)$.

The core of this paper is devoted to prove that Eqs. (1.10) have a solution $A_{\mu}(t) \in C^{\infty}\left(R^{3}\right)$ and $\psi(t) \in W^{\infty, 2}\left(R^{3}, C^{4}\right),{ }^{1} t \geqq 0$, for every final state $(a, \dot{a}, \alpha) \in E_{+}$ being sufficiently small (Theorem 2.4). The method of proof, which is close to the one used in [11], consists of first constructing (Theorem 2.3) explicitly an approximate solution of Eqs. (1.10) absorbing the most slowly decaying (in spacetime) parts of $A_{\mu}(t)$ and $\psi(t)$. When the approximate solution has been established it is a simple matter, using a priori time decay of the remaining part of the solution, to prove the existence for Eq. (1.10) (Theorem 2.4). It is proved that the map $\Omega:(a, \dot{a}, \alpha) \rightarrow(A(0), \dot{A}(0), \psi(0))$ intertwines the nonlinear and the linear evolutions (Theorem 2.7).

\section{Statement and Proof of the Main Results}

The proof of the results of this paper is based on the existence of an approximate solution of Eqs. (1.10). We postpone the proof of this fact to Sect. 3.

Let the phase functions $S_{\varepsilon} \in C^{1}\left(R^{+}, C^{\infty}\left(R^{3}, R\right)\right), \varepsilon= \pm$ satisfy

$$
\begin{gathered}
\left|\nabla_{k}^{n}\left(S_{\varepsilon}(k, t)-\varepsilon \omega(k) t\right)\right| \leqq C_{n} \log (2+t), \\
\left|\nabla_{k}^{n}\left(\frac{\partial}{\partial t} S_{\varepsilon}(k, t)-\varepsilon \omega(k)\right)\right| \leqq C_{n}(1+t)^{-1},
\end{gathered}
$$

for all $t \geqq 0, k \in R^{3}, n \geqq 0$ and some constants $C_{n}<\infty$.

Let the map $t \rightarrow(\overline{\beta(} t))^{\wedge} \in \mathscr{D}\left(R^{3}, C^{4}\right)$ be $C^{1}$ and let $\beta(t)=\beta_{+}(t)+\beta_{-}(t)$ satisfy $^{2}$

$$
\begin{gathered}
\left|\nabla_{k}^{n}\left(\left(\beta_{\varepsilon}(t)\right)^{\wedge}(k)-\hat{\alpha}_{\varepsilon}(k)\right)\right| \leqq C_{n}(1+t)^{-1}, \\
\left|\nabla_{k}^{n} \frac{\partial}{\partial t}\left(\beta_{\varepsilon}(t)\right)^{\wedge}(k)\right| \leqq C_{n}(1+t)^{-2}, \\
\operatorname{supp}(\beta(t))^{\wedge} \subset K
\end{gathered}
$$

for all $t \geqq 0, k \in R^{3}, n \geqq 0, \varepsilon= \pm$, and for some $\hat{\alpha}_{\varepsilon}=P_{\varepsilon} \hat{\alpha}_{\varepsilon} \in \mathscr{D}\left(R^{3}, C^{4}\right)$, some constants $C_{n}<+\infty$ and some compact set $K \subset R^{3}$.

Throughout this paper $\phi$ will be defined by:

$$
\left(\phi_{\varepsilon}(t)\right)^{\wedge}(k)=e^{i S_{\varepsilon}(k, t)}\left(\beta_{\varepsilon}(t)\right)^{\wedge}(k), \quad \phi=\phi_{+}+\phi_{-} .
$$

The following theorem, stating that $\phi$ has decrease properties similar to those of solutions of the free Dirac equation, will be proved in Appendix A:

Theorem 2.1. Let conditions (2.1) and (2.2) be satisfied. Then $\|\phi(t)\|_{W^{n, \infty}}$ $\leqq C_{n}(1+t)^{-3 / 2}$ for all $t \geqq 0, n \geqq 0$ and some $C_{n}<\infty$.

${ }^{1} W^{n, p}\left(R^{m}, C^{k}\right)$ is the subspace of $L^{p}\left(R^{m}, C^{k}\right)$ for which

$$
\|\mathrm{f}\|_{W^{n, p}}=\sum_{|\alpha| \leqq n}\left\|\partial^{\alpha} f\right\|_{L^{p}}<\infty, \quad W^{\infty, p}=\bigcup_{n \geqq 0} W^{n, p}
$$

${ }^{2}$ In general $\beta_{\varepsilon}$ will not be equal to $P_{\varepsilon}(\partial) \beta_{\varepsilon}$ 
By a long range electromegnetic potential, we mean a function

$$
G \in C^{1}\left(R^{+}, C^{\infty}\left(R^{3}, R^{4}\right)\right)
$$

satisfying

$$
\begin{gathered}
|k|^{2}|k t|^{n}\left|\left(G_{\mu}(t)\right)^{\wedge}(k)\right| \leqq C_{n}, \\
|k||k t|^{n} \mid\left(\frac{d}{d t} G_{\mu}(t) \hat{)}(k) \mid \leqq C_{n},\right. \\
\operatorname{supp}\left(G_{\mu}(t)\right)^{\wedge} \subset K,
\end{gathered}
$$

for all $t \geqq 0, k \in R^{3}, \mu=0,1,2,3, n=0,1, \ldots$ and some compact $K$ independent of $t$ and some finite constants $C_{n}$.

It follows from (2.4) that (with new $C_{n}$ ):

$$
\begin{gathered}
\left\|G_{\mu}(t)\right\|_{W^{n, \infty}} \leqq C_{n}(1+t)^{-1}, \\
\left\|(d / d t) G_{\mu}(t)\right\|_{W^{n, \infty}} \leqq C_{n}(1+t)^{-2},
\end{gathered}
$$

for all $n \geqq 0, \mu=0,1,2,3$ and some $C_{n}<\infty$.

We can now state what is meant by an approximate solution of Eq. (1.10).

Definition 2.2. Let $(a, \dot{a}, \alpha) \in E_{+}$and let $S, \beta, G$ satisfy respectively (2.1), (2.2), and (2.4). $(B, \phi)$ is said to be an approximate solution of (1.10) if $B_{\delta}(t)=\dot{\mu}(t) a_{\delta}+\mu(t) \dot{a}_{\delta}$ $+G_{\delta}(t)$ and

i)

ii)

iii)

$$
\begin{gathered}
\left\|M_{\delta}(t)\right\|_{W^{n, 2}} \leqq C_{n}(1+t)^{-3 / 2}, \\
\left\|(d / d t) M_{\delta}(t)\right\|_{W^{n, 2}} \leqq C_{n}(1+t)^{-3 / 2}, \\
\|\Phi(t)\|_{W^{n, 2}} \leqq C_{n}(1+t)^{-2},
\end{gathered}
$$

for all $t \geqq 0, n \geqq 0$, and $0 \leqq \delta \leqq 3$. Here

$$
M_{\delta}(t)=\dot{\mu}(t) a_{\delta}+\mu(t) \dot{a}_{\delta}+\int_{\infty}^{t} d s \mu(t-s) \overline{\phi(s)} \gamma_{\delta} \phi(s)-B_{\delta}(t)
$$

and

$$
\Phi(t)=W_{0}(t, 0) \alpha+\int_{\infty}^{t} d s W_{0}(t, s)\left(\mathscr{D}-i \gamma^{0} \gamma^{\mu} B_{\mu}(s)-\mathscr{L}(s)\right) \phi(s)-\phi(t)
$$

A constructive proof of the existence of approximate solutions will be given in Sect. 3 .

Theorem 2.3. For every $(a, \dot{a}, \alpha) \in E_{+}$there exists an approximate solution $(B, \phi)$ of Eq. (1.10). Further $(B, \phi)$ can be chosen such that the constants (in Definition 2.2) $C_{n} \rightarrow 0$ as $(a, \dot{a}, \alpha) \rightarrow 0$ in $E_{+}$.

Let $E_{+}\left(\delta_{1}, \delta_{2}\right)$, where $0<\delta_{1}<\delta_{2}$, denote the subspace of functions $(a, \dot{a}, \alpha) \in E_{+}$ such that $\operatorname{supp} \hat{a} \subset\left\{\delta_{1} \leqq|k| \leqq \delta_{2}\right\}$, supp $\hat{\dot{a}} \subset\left\{\delta_{1} \leqq|k| \leqq \delta_{2}\right\}$ and $\operatorname{supp} \hat{\alpha} \subset\left\{|k| \leqq \delta_{2}\right\}$. With Gross [8] we introduce the Hilbert spaces $M_{n}, n \geqq 0$, of distributions $(f, g) \in S^{\prime}\left(R^{3}, R^{4}\right) \oplus S^{\prime}\left(R^{3}, R^{4}\right)$ satisfying

$$
\|(f, g)\|_{M_{n}}=\left\|(1-\Delta)^{n / 2-1 / 4} \nabla f\right\|_{L^{2}}+\left\|(1-\Delta)^{n / 2-1 / 4} g\right\|_{L^{2}}<\infty .
$$


$E$ is the space of elements $(f, g, \chi)$ such that $(f, g) \in M_{n}$ and $\chi \in W^{n, 2}\left(R^{3}, C^{4}\right)$ for all $n \geqq 0$.

The main result of this paper can now be stated:

Theorem 2.4. Given $0<\delta_{1}<\delta_{2}$ there is an open set $\mathcal{O}\left(\delta_{1}, \delta_{2}\right)$ in $E_{+}\left(\delta_{1}, \delta_{2}\right)$ such that, if $(a, \dot{a}, \alpha) \in \mathcal{O}\left(\delta_{1}, \delta_{2}\right)$, then for $\mathscr{L}$ given by Theorem 2.3 , the equations

$$
\begin{gathered}
A_{v}(t)=\dot{\mu}(t) a_{v}+\mu(t) \dot{a}_{v}+\int_{\infty}^{t} \mu(t-s) \overline{\psi(s)} \delta_{v} \psi(s) d s, \\
\psi(t)=W_{0}(t, 0) \alpha+\int_{\infty}^{t} W_{0}(t, s)\left(\mathscr{D}-i \gamma^{0} \gamma^{\mu} A_{\mu}(s)-\mathscr{L}(s)\right) \psi(s) d s,
\end{gathered}
$$

where $v=0,1,2,3$ and $t \geqq 0$, have a unique solution $(A, \psi)$ with $(A, \dot{A}, \psi) \in C^{\infty}\left(R^{+}, E\right)$. The solution satisfies the following decay property:

$$
\begin{aligned}
& \sup _{t \geqq 0}\left(\|(A(t),(d / d t) A(t))\|_{M_{n}}+\|\psi(t)\|_{W^{n, 2}}+(1+t)\|A(t)\|_{W^{n, \infty}}\right. \\
& \left.\quad+(1+t)^{3 / 2}\|\psi(t)\|_{W^{n, \infty}}\right)<+\infty
\end{aligned}
$$

for every $n \geqq 0$. The solution $(A, \dot{A}, \psi)$ depends continuously on the data $(a, \dot{a}, \alpha)$.

Proof. Let $D_{n}, n \geqq 0$ be the space of continuous functions from $R^{+}$to

$$
W^{n, 2}\left(R^{3}, R^{4}\right) \oplus W^{n, 2}\left(R^{3}, C^{4}\right), \quad t \rightarrow\left(b_{\mu}(t), \varphi(t)\right),
$$

where $\mu=0,1,2,3$ satisfying

$$
\|(b, \varphi)\|_{D_{n}}=\sup _{t \leqq 0}\left((1+t) \sum_{\mu}\left\|b_{\mu}(t)\right\|_{W^{n, 2}}+(1+t)^{3 / 2}\|\varphi(t)\|_{W^{n, 2}}\right)<\infty .
$$

Given an open neighbourhood $\mathcal{O}\left(\delta_{1}, \delta_{2}\right)$ of zero in $E_{+}\left(\delta_{1}, \delta_{2}\right)$ there exists according to Theorem 2.3 an approximate solution $(B, \phi)$ for every $(a, \dot{a}, \alpha) \in \mathcal{O}\left(\delta_{1}, \delta_{2}\right)$.

Introduce the function $\mathscr{K}:(b, \varphi) \rightarrow\left(b^{\prime}, \varphi^{\prime}\right),(b, \varphi) \in D_{n}$ for some fixed $n \geqq 2$, by

$$
\begin{aligned}
b_{\delta}^{\prime}(t)= & M_{\delta}(t)+\int_{\infty}^{t} \mu(t-s)\left(\overline{\varphi(s)} \gamma_{\delta} \varphi(s)+\overline{\varphi(s)} \gamma_{\delta} \phi(s)+\overline{\phi(s)} \gamma_{\delta} \varphi(s)\right) d s, \\
\varphi^{\prime}(t)= & \Phi(t)+\int_{\infty}^{t} W_{0}(t, s)\left(\left(\mathscr{D}-i \gamma^{0} \gamma^{\mu} B_{\mu}(s)-\mathscr{L}(s)\right) \varphi(s)\right. \\
& \left.-i \gamma^{0} \gamma^{\mu} b_{\mu}(s) \varphi(s)-i \gamma^{0} \gamma^{\mu} b_{\mu}(s) \phi(s)\right) d s,
\end{aligned}
$$

where $M$ is given by (2.6) and $\Phi$ by (2.7). $\mathscr{K}$ is a function from $D_{n}$ to $D_{n}$ if $n \geqq 2$. In fact, $b^{\prime}(t)$ and $\varphi(t)$ are continuous in $t$ and

$$
\begin{aligned}
\left\|b_{\delta}^{\prime}(t)\right\|_{W^{n, 2}} \leqq & \left\|M_{\delta}(t)\right\|_{W^{n, 2}}+C_{n} \int_{t}^{\infty}(s-t)\left(\|\varphi(s)\|_{W^{n, 2}}^{2}+\|\varphi(s)\|_{W^{n, 2}}\|\phi(s)\|_{W^{n, \infty}}\right) d s, \\
\left\|\varphi^{\prime}(t)\right\|_{W^{n, 2}} \leqq & \|\Phi(t)\|_{W^{n, 2}}+C_{n} \int_{t}^{\infty}\left(\left\|\mathscr{D}-i \gamma^{0} \gamma^{\mu} B_{\mu}(s)-\mathscr{L}(s)\right\|_{L^{\left(W^{n, 2}\right)}}\|\varphi(s)\|_{W^{n, 2}}\right. \\
& \left.\quad\left\|b_{\mu}(s)\right\|_{W^{n, 2}}\|\varphi(s)\|_{W^{n, 2}}+\left\|b_{\mu}(s)\right\|_{W^{n, 2}}\|\phi(s)\|_{W^{n, \infty}}\right) d s .
\end{aligned}
$$

Using the fact that $(b, \varphi) \in D_{n}$, introducing $\varrho_{1}=\|(M, \Phi)\|_{D_{n}}$,

$$
\varrho_{2}=\sup _{t \geqq 0}\left((1+t)^{3 / 2}\|\phi(t)\|_{W^{n, \infty}}\right)
$$


and

$$
\varrho_{3}=\sup _{t \geqq 0}\left((1+t)\left\|\mathscr{D}-i \gamma^{0} \gamma^{\mu} B_{\mu}(t)-\mathscr{L}(t)\right\|_{L\left(W^{n, 2}\right)}\right),
$$

which, according to Theorem 2.3, Definition 2.2 and formula (2.1b) are finite, we get

$$
\begin{aligned}
\left\|b_{\mu}^{\prime}(t)\right\|_{W^{n, 2}} \leqq & \left\|M_{\mu}(t)\right\|_{W^{n, 2}}+C_{n}(1+t)^{-1}\left(\|(b, \varphi)\|_{D_{n}}^{2}+\|(b, \varphi)\|_{D_{n}} \varrho_{2}\right) \\
\left\|\varphi^{\prime}(t)\right\|_{W^{n, 2}} \leqq & \Phi(t) \|_{W^{n, 2}}+C_{n}(1+t)^{-3 / 2}\left(\|(b, \varphi)\|_{D_{n}} \varrho_{3}\right. \\
& \left.+\|(b, \varphi)\|_{D_{n}}^{2}+\|(b, \varphi)\|_{D_{n}} \varrho_{2}\right) .
\end{aligned}
$$

The last inequalities give

$$
\left\|\left(b^{\prime}, \varphi^{\prime}\right)\right\|_{D_{n}} \leqq \varrho_{1}+C_{n}\left(\|(b, \varphi)\|_{D_{n}}^{2}+\|(b, \varphi)\|_{D_{n}}\left(\varrho_{2}+\varrho_{3}\right)\right),
$$

which shows that $\mathscr{K}: D_{n} \rightarrow D_{n}$ for every $n \geqq 2$. (Here $C_{n}$ depends only on the definition of the norms).

Similarly one gets:

$$
\begin{aligned}
\left\|\mathscr{K}\left(b_{1}, \varphi_{1}\right)-\mathscr{K}\left(b_{2}, \varphi_{2}\right)\right\|_{D_{n}} \leqq & C_{n}\left(( \| ( b _ { 1 } , \varphi _ { 1 } ) \| _ { D _ { n } } + \| ( b _ { 2 } , \varphi _ { 2 } ) \| _ { D _ { n } } ) \left(\left\|\left(b_{1}, \varphi_{1}\right)-\left(b_{2}, \varphi_{2}\right)\right\|_{D_{n}}\right.\right. \\
& \left.+\left(\varrho_{2}+\varrho_{3}\right)\left\|\left(b_{1}, \varphi_{1}\right)-\left(b_{2}, \varphi_{2}\right)\right\|_{D_{n}}\right),
\end{aligned}
$$

which shows that $\mathscr{K}$ is a contraction in a sufficiently small neighbourhood of $O$ in $D_{n}$ if $\varrho_{2}+\varrho_{3}$ is sufficiently small. $n$ being fixed we choose, according to Theorem 2.3, $\mathcal{O}\left(\delta_{1}, \delta_{2}\right)$ so that $\varrho_{2}+\varrho_{3}$ is sufficiently small. The equation

$$
(b, \varphi)=\mathscr{K}(b, \varphi)
$$

has then a unique solution $(b, \varphi) \in D_{n}$.

It follows now, taking $\mathcal{O}\left(\delta_{1}, \delta_{2}\right)$ sufficiently small, that $(b, \varphi) \in D_{1}$ for all $i \geqq 0$. In fact, omitting the details, if $\left(\nabla^{k} b, \nabla^{k} \varphi\right) \in D_{n}$ for $k=0,1, \ldots, m$, then $\left(\nabla^{m+1} b, \nabla^{m+1} \varphi\right)$ has to satisfy the equation

$$
\left(\nabla^{m+1} b, \nabla^{m+1} \varphi\right)=F_{m+1}+L\left(\nabla^{m+1} b, \nabla^{m+1} \varphi\right),
$$

where $L$ is a real linear operator on $D_{n}$, independent of $m$, with $\|L\|<1$ if $\mathcal{O}\left(\delta_{1}, \delta_{2}\right)$ is sufficiently small. $F_{m+1} \in D_{n}$ and $F_{m+1}$ depends only on $\left(\nabla^{k} b, \nabla^{k} \varphi\right)$ for $k=0, \ldots, m$. Hence by induction

$$
\left(\nabla^{k} b, \nabla^{k} \varphi\right) \in D_{n} \text { for all } m \geqq 0 .
$$

It follows from Eq. (2.9) that the solution $(b, \varphi) \in \bigcup_{m \geqq 0} D_{m}$ satisfies

$$
\left.\frac{d}{d t} b_{v}(t)=\frac{d}{d t} M_{v}(t)+\int_{\infty}^{t} \dot{\mu}(t-s) \overline{(\varphi(s)} \gamma_{v} \varphi(s)+\overline{\varphi(s)} \gamma_{v} \phi(s)+\overline{\phi(s)} \gamma_{v} \varphi(s)\right) d s .
$$

This equation, Theorem 2.3 and $(b, \varphi) \in \bigcup_{m \geqq 0} D_{m}$ give that

$$
\left\|(d / d t) b_{v}(t)\right\|_{W^{m, 2}} \leqq C_{m}(1+t)^{-3 / 2}
$$

for all $m \geqq 0$. In particular we conclude that $(b(t), \dot{b}(t)) \in \bigcup \begin{aligned} & \bigcup \\ & M_{m}\end{aligned}$ for all $t \geqq 0$ and that $(b(t), \dot{b}(t), \varphi(t))$ is uniformly bounded in $E$ for $t \geqq 0$. 
We define $A=B+b$ and $\psi=\phi+\varphi$. It follows from Definition 2.2 and inequalities (2.4) and (2.5a) that

$$
\sup _{t \geqq 0}\left(\|\left(B(t), \dot{B}(t)\left\|_{M_{n}}+(1+t)\right\| B(t) \|_{W^{n, \infty}}\right)<+\infty \quad \text { for all } n \geqq 0 .\right.
$$

It follows from Theorem 2.1 that

$$
\sup _{t \geqq 0}\left(\|\phi(t)\|_{W^{n, 2}}+(1+t)^{3 / 2}\|\phi(t)\|_{W^{n, \infty}}\right)<+\infty \quad \text { for all } n \geqq 0 .
$$

Together with the above properties of $(b, \varphi)$ this shows that the decay announced in the theorem is true.

By the construction of $\mathscr{K}, A$, and $\psi$, Eq. (2.8) is satisfied by $(A, \psi)$ with $(A, \dot{A}, \psi) \in C^{0}\left(R^{+}, E\right)$. For a $\mathscr{L}$ given by Theorem 2.3 the solution $(A, \psi)$ is unique as $\mathscr{K}$ has been proved to be a contraction. The fact that $(A, \dot{A}, \psi) \in C^{\infty}\left(R^{+}, E\right)$ follows from differentiation in $t$ of Eq. (2.8). Q.E.D.

The solution $(A, \psi)$ of Eq. (2.8) satisfies the M-D equations. The proof of this fact being obvious we only state the result:

Corollary 2.5. In the situation of Theorem 2.4, $A \in C^{\infty}\left(R^{+} \times R^{3}, R^{4}\right)$, $\psi \in C^{\infty}\left(R^{+} \times R^{3}, C^{4}\right)$, and $(A, \psi)$ satisfies Eq. (1.1a) and (1.1b).

If the data $(a, \dot{a}, \psi)$ satisfy the Lorentz gauge condition then this is also the case for the solution: Corollary 2.6. If $\dot{a}^{0}+\sum_{i=1}^{3} \partial_{i} a^{i}=0$ and $\Delta a^{0}+\sum_{i=1}^{3} \partial_{i} \dot{a}^{i}=0$, then $A$ in Corollary 2.5
satisfies $\partial_{\mu} A^{\mu}=0$.

Proof. Equations (1.1b) and (2.8a) give that

$$
\left(\partial_{\mu} A^{\mu}\right)(t)=\mu(t)\left(\Delta a^{0}+\sum_{i=1}^{3} \partial_{1} \dot{a}^{1}\right)+\dot{\mu}(t)\left(\dot{a}^{0}+\sum_{i=1}^{3} \partial_{i} a^{1}\right) \text {. Q.E.D. }
$$

Let $\mathcal{O}$ be a union of sets $\mathcal{O}\left(\delta_{1}, \delta_{2}\right)$ given by Theorem 2.4. Denoting $d / d t A(t)$ $=\dot{A}(t)$, we define a modified wave operator $\Omega: \mathcal{O} \rightarrow E$ for Eq. (1.2) by $\Omega(a, \dot{a}, \alpha)$ $=(A(0), \dot{A}(0), \psi(0))$. Let

$$
U^{1}(t)=\exp \left(\left(\begin{array}{ll}
0 & 1 \\
\Delta & 0
\end{array}\right) t\right) \oplus \exp (\mathscr{D} t)
$$

be the evolution operator defined by the linear part of Eq. (1.2). That $\Omega$ intertwine the linear and nonlinear evolution is the content of the following theorem:

Theorem 2.7. The continuous map $\Omega: \mathcal{O} \rightarrow$ E satisfies

$$
(A(t), \dot{A}(t), \psi(t))=\Omega\left(U_{t}^{1}(a, \dot{a}, \alpha)\right), \quad t \geqq 0,
$$

where $(a, \dot{a}, \alpha) \in \mathcal{O}$ and $(A, \psi)$ is the solution of Eq. (2.8) given by Theorem 2.4.

Proof. Let $r \geqq 0$, let $(A, \psi)$, be the solution of Eq. (2.8) with initial data $(a, \dot{a}, \alpha) \in \mathcal{O}$ and let $\left(A^{\prime}, \psi^{\prime}\right)$ be the solution of Eq. (2.8) with initial data $U_{r}^{1}(a, \dot{a}, \alpha)$, i.e.

$$
A_{v}^{\prime}(t)=\dot{\mu}(t+r) a_{v}+\mu(t+r) \dot{a}_{v}+\int_{\infty}^{t} \mu(t-s) \overline{\psi^{\prime}(s)} \gamma_{v}(s) \psi^{\prime}(s) d s
$$


and

$$
\psi^{\prime}(t)=W_{0}^{\prime}(t, 0) e^{\mathscr{D} r} \alpha+\int_{\infty}^{t} W_{0}^{\prime}(t, s)\left(\mathscr{D}-i \gamma^{0} \gamma^{v} A_{v}^{\prime}(s)-\mathscr{L}^{\prime}(s)\right) \psi^{\prime}(s) d s,
$$

where $W_{0}^{\prime}$ and $\mathscr{L}^{\prime}$ are given by Theorem 2.3 for the initial data $U_{r}^{1}(a, \dot{a}, \alpha)$. It is enough to prove that $A^{\prime}(t)=A(t+r)$ and that $\psi^{\prime}(t)=\psi(t+r)$ for all $t \geqq 0$.

Equation (2.8b) gives, for $t, r \geqq 0$,

$$
\psi(t+r)=W_{0}(t+r, 0) \alpha+\int_{\infty}^{t} W_{0}(t+r, s+r)\left(\mathscr{D}-i \gamma^{0} \gamma^{v} A_{v}(s+r)-\mathscr{L}(s+r)\right) \psi(s+r) d s .
$$

It follows from this equation that $\lim _{t \rightarrow \infty} W_{0}^{\prime}(0, t) \psi(t+r)=e^{\mathscr{D} r} \alpha$, where the limit is taken in $W^{n, 2}$ for any $n \geqq 0$. Equation (1.2b) gives:

$$
\begin{aligned}
(d / d t)\left(W_{0}^{\prime}(0, t) \psi(t+r)\right)= & -W_{0}^{\prime}(0, t) \mathscr{L}^{\prime}(t) \psi(t+r) \\
& +W_{0}^{\prime}(0, t)\left(\mathscr{D}-i A_{\mu}(t+r) \gamma_{\gamma}^{0} \gamma^{\mu}\right) \psi(t+r) .
\end{aligned}
$$

This equation can be integrated from $+\infty$ to $t$, and we can conclude that Eq. (2.14) is satisfied with $A^{\prime}(t)=A(t+r)$ and $\psi^{\prime}(t)=\psi(t+r)$. This proves the theorem, as the solution of (2.14) is unique according to Theorem 2.4. Q.E.D.

\section{Construction of an Approximate Solution}

In this paragraph, we construct approximate solutions of the $\mathrm{M}-\mathrm{D}$ equations, which satisfy Definition 2.2.

Introduce

$$
Q_{T}^{v}(S, B, t)=\int_{T}^{t} \mu(t-s) \overline{\phi(s)} \gamma^{v} \phi(s) d s,
$$

where $T \geqq t \geqq 0$ and $\phi$ is given by (2.3). If there is no risk of confusion we write $Q_{T}^{v}(t)$ instead of $Q_{T}^{v}(S, \beta, t)$. Denote by $Q_{\infty}^{v}(t)=Q^{v}(t)$ and by

$$
Q(t)=\left(Q^{0}(t), Q^{1}(t), Q^{2}(t), Q^{3}(t)\right) .
$$

To begin with we prove existence and decay properties of $Q(t)$. We define

$$
J_{\varepsilon}^{\mu}(t)=\overline{\phi_{\varepsilon}(t)} \gamma^{\mu} \phi_{\varepsilon}(t), \quad \varepsilon= \pm .
$$

When needed the dependence on $S$ and $\beta$ will be indicated by $J_{\varepsilon}^{\mu}(t)=J_{\varepsilon}^{\mu}(S, \beta, t)$.

Lemma 3.1. There are finite constants $C_{n}$ such that

$$
(|k| t)^{n}\left|\left(J_{\varepsilon}^{\mu}(t)\right)^{\wedge}(k)\right| \leqq C_{n}
$$

for all $k \in R^{3}, t \geqq 0, \varepsilon= \pm$ and integers $n \geqq 0$. The support of $\left(J_{\varepsilon}^{\mu}(t)\right)^{\wedge}$ is contained in a compact set independent of $t$.

Proof. Introduce, for given $\varepsilon$ and $\mu$,

$$
u\left(k, k^{\prime}, t\right)=\overline{\left(\left(\beta_{\varepsilon}(t)\right)^{\wedge}\right)\left(k^{\prime}-k\right)} \gamma^{\mu}\left(\beta_{\varepsilon}(t)\right)^{\wedge}\left(k^{\prime}\right)
$$

and

$$
v\left(k, k^{\prime}, t\right)=S_{\varepsilon}\left(k^{\prime}, t\right)-S_{\varepsilon}\left(k^{\prime}-k, t\right) .
$$


As $\operatorname{supp}\left(\beta_{\varepsilon}(t)\right)^{\wedge}$ is contained in a compact set independent of $t$, there is $\delta>0$ such that

$$
\operatorname{supp}\left(J_{\varepsilon}^{\mu}(t)\right)^{\wedge} \subset B(0, \delta) \text { for all } t \geqq 0 .
$$

Here $B(0, \delta)=\left\{k \in R^{3}|| k \mid<\delta\right\}$. Let $0<\delta_{1}<\delta$. If $|k|>\delta$, then (3.3) is satisfied as $\left(J_{\varepsilon}^{\mu}(t)\right)^{\wedge}(k)=0$ for $t \geqq 0$.

Let $\delta>|k|>\delta_{1}$. Then

$$
\nabla_{k^{\prime}}\left(\omega\left(k^{\prime}\right)-\omega\left(k-k^{\prime}\right)\right)=\frac{k^{\prime}}{\omega\left(k^{\prime}\right)}-\frac{k^{\prime}-k}{\omega\left(k^{\prime}-k\right)}=0
$$

has no solution. Hence there is $v>0$ such that

$$
\left|\nabla_{k^{\prime}}\left(\omega\left(k^{\prime}\right)-\omega\left(k^{\prime}-k\right)\right)\right| \geqq 2 v \quad \text { for all } k^{\prime} \in \operatorname{supp}\left(\beta_{\varepsilon}(t)\right)^{\wedge} \subset K,
$$

and $\delta_{1}<|k|<\delta$. If $t$ is sufficiently large, it then follows from (2.1a) that $\left|\nabla_{k^{\prime}} v\left(k, k^{\prime}, t\right) / t\right| \geqq v$ for $k^{\prime} \in K$ and $\delta_{1}<|k|<\delta$. Theorem 7.7.1 of [9] and (2.2a) now give $|k t|^{n}\left|\left(J_{\varepsilon}^{\mu}(t)\right)^{\wedge}(k)\right| \leqq C_{n}$ for all $n \geqq 0$. Let $|k| \leqq \delta_{1}$ and introduce $h\left(k, k^{\prime}, t\right): R^{3} \rightarrow R$ by

$$
h\left(k, k^{\prime}, t\right)=\int_{0}^{1} \nabla_{k}\left(t^{-1} v\left(s k, k^{\prime}, t\right)\right) d s .
$$

One gets by the definition of $h$ that

$$
v\left(k, k^{\prime}, t\right)=h\left(k, k^{\prime}, t\right) \cdot k t,
$$

We observe that the matrix, with elements

$$
\left(\nabla_{k^{\prime}} h\left(0, k^{\prime}, t\right)\right)_{i j}=\frac{1}{t} \frac{\partial^{2}}{\partial k_{i} \partial k_{j}} S_{\varepsilon}\left(k^{\prime}, t\right),
$$

where $1 \leqq i \leqq 3,1 \leqq j \leqq 3$, is, according to $(2.1 \mathrm{a})$, uniformly bounded together with its inverse for all $k^{\prime}$ if $t$ is sufficiently large. This shows that $\nabla_{k^{\prime}} h\left(k, k^{\prime}, t\right)$ is non-singular for all $|k| \leqq \delta_{1}, k \in K$, and $t \geqq T$, if $\delta_{1}$ is sufficiently small and $T$ sufficiently large. As $\nabla_{k^{n}}^{n} h\left(k, k^{\prime}, t\right)$ is bounded for all $t$ and $k, k^{\prime} \in R^{3}$, it follows from the implicit functions theorem and by partial integration that

$$
(|k| t)^{n}\left|\int d k^{\prime} u\left(k, k^{\prime}, t\right) e^{i h\left(k, k^{\prime}, t\right) \cdot k t}\right| \leqq C_{n}, \quad n \geqq 0
$$

for all $|k| \leqq \delta_{1}$ and $t \geqq 0$ [we also used that $\nabla_{k^{\prime}}^{n} u\left(k, k^{\prime}, t\right)$ is bounded by $(2.2 \mathrm{a})$ and $\left.\hat{\beta}_{\varepsilon} \in \mathscr{D}\left(R^{3}, C^{4}\right)\right]$. Q.E.D.

Let $\tau_{i}, i=1,2$ be monotonic continuous functions $\tau_{i}: R^{+} \rightarrow R^{+}$with

$$
\tau_{1}(t) \leqq C(\log (1+t)+1) \quad \text { and } \quad \tau_{2}(t) \leqq C(1+t)^{-1} \quad \text { for some } \quad C \geqq 0 .
$$

Lemmma 3.2. Let $S^{(1)}$ and $S^{(2)}$ satisfy (2.1a) and let $\beta^{(1)}$ and $\beta^{(2)}$ satisfy the conditions (2.2a) and (2.2c). If, for some constants $C_{n} \geqq 0$,

$$
\left|\nabla_{k}^{n}\left(S_{\varepsilon}^{(1)}(k, t)-S_{\varepsilon}^{(2)}(k, t)\right)\right| \leqq C_{n} \tau_{1}(t) \quad \text { and } \quad\left|\nabla_{k}^{n}\left(\beta_{\varepsilon}^{(1)}(t)-\beta_{\varepsilon}^{(2)}(t)\right)^{\wedge}(k)\right| \leqq C_{n} \tau_{2}(t),
$$

then there are constants $C_{n}^{\prime}$ depending only on $\left\{C_{l}\right\}_{l=0}^{\infty}$ such that

$$
|k t|^{n}\left|\left(J_{\varepsilon}^{\mu}\left(S^{(1)}, \beta^{(1)}, t\right)\right)^{\wedge}(k)-\left(J_{\varepsilon}^{\mu}\left(S^{(2)}, \beta^{(2)}, t\right)\right)^{\wedge}(k)\right| \leqq C_{n}^{\prime}\left(\tau_{1}(t)(1+t)^{-1}+\tau_{2}(t)\right)
$$

for all $\varepsilon= \pm, k \in R^{3}, t \geqq 0$ and integers $n \geqq 0$. 
The proof of this lemma, being very similar to that of Lemma 3.1, we omit it. Introduce

$$
I_{\varepsilon}^{\mu}(t)=\overline{\phi_{-\varepsilon}(t)} \gamma^{\mu} \phi_{\varepsilon}(t)
$$

where $\varepsilon= \pm 1, \mu=0,1,2,3$ and $t \geqq 0$. When needed the dependence on $\beta$ and $S$ will be indicated by $I_{\varepsilon}^{\mu}(S, \beta, t)$.

Lemma 3.3. There is a finite constant $C$ such that

$$
\left|\left(I_{\varepsilon}^{\mu}(t)\right)^{\wedge}(k)\right| \leqq C(1+t)^{-3 / 2}
$$

and

$$
\left|\frac{\partial}{\partial t}\left(e^{-2 i \varepsilon \omega(k / 2) t}\left(I_{\varepsilon}^{\mu}(t)\right)^{\wedge}(k)\right)\right| \leqq C(1+t)^{-5 / 2}
$$

for all $\varepsilon= \pm, t \geqq 0$, and $\mu=0,1,2,3$. The support of $\left(I_{\varepsilon}^{\mu}(t)\right)^{\wedge}$ is contained in a compact set independent of $t \geqq 0$.

Proof. The existence of a compact set $K_{1}$ such that $\operatorname{supp}\left(I_{\varepsilon}^{\mu}(t)\right)^{\wedge} \subset K_{1}$, for all $t \geqq 0$ is a direct consequence of the formula (3.8) and the property (2.2c) of $\beta$.

Denote, for given $\varepsilon$ and $\mu$,

$$
u\left(k, k^{\prime}, t\right)=\left(\left(\beta_{-\varepsilon}(t)\right)^{\wedge}\left(k^{\prime}-k\right) \gamma^{\mu}\left(\beta_{\varepsilon}(t)\right)^{\wedge}\left(k^{\prime}\right)\right.
$$

and

$$
v\left(k, k^{\prime}, t\right)=S_{\varepsilon}\left(k^{\prime}, t\right)-S_{-\varepsilon}\left(k^{\prime}-k, t\right)
$$

Then

$$
\left(I_{\varepsilon}^{\mu}(t)\right)^{\wedge}(k)=\int d k^{\prime} u\left(k, k^{\prime}, t\right) e^{i v\left(k, k^{\prime}, t\right)} .
$$

By the implicit functions theorem, equation $t^{-1} \nabla_{k^{\prime}} v\left(k, k^{\prime}, t\right)=0$ has for $k \in K_{1}$ and $t$ sufficiently big a unique solution $k^{\prime}=p(k, t)$, which is close to $k / 2$. In fact, the unique solution of $\nabla_{k^{\prime}}\left(\omega\left(k^{\prime}\right)+\omega\left(k^{\prime}-k\right)\right)=0$ is $k^{\prime}=\frac{1}{2} k$ and the determinant of $\frac{\partial^{2}}{\partial k_{i}^{\prime} \partial k_{j}^{\prime}}\left(\omega\left(k^{\prime}\right)+\omega\left(k^{\prime}-k\right)\right)$ for $k^{\prime}=\frac{1}{2} k$ is $8 m^{2} /\left(\omega\left(\frac{1}{2} k\right)\right)^{5}$. By continuity the determinant of $\left(\partial^{2} /\left(\partial k_{i}^{\prime} \partial k_{j}^{\prime}\right)\right) v\left(k, k^{\prime}, t\right)$ is different from zero in a sufficiently small neighbourhood of $k^{\prime}=\frac{1}{2} k$, if $t$ is big enough, which proves the existence of the solution $k^{\prime}=p(k, t)$. $p$ is a continuous function and $k \rightarrow p(k, t)$ and $k \rightarrow(\partial / \partial k) p(k, t)$ are $C^{\infty}$. It follows from the equation $\nabla_{k^{\prime}} v\left(k, k^{\prime}, t\right)=0$ and (2.1) that

$$
\left|p(k, t)-\frac{1}{2} k\right| \leqq C(1+t)^{-1} \log (2+t) \quad \text { and } \quad\left|\frac{\partial}{\partial t} p(k, t)\right| \leqq C(1+t)^{-2} \log (2+t)
$$

for all $k \in K_{1}$ and $t \geqq 0$. Consequently

$$
\left|t^{-1} v(k, p(k, t), t)-\varepsilon 2 \omega(k / 2)\right| \leqq C(1+t)^{-1} \log (2+t)
$$

and

$$
\left|\frac{\partial}{\partial t} t^{-1} v(k, p(k, t), t)\right| \leqq C(1+t)^{-2} \log (2+t)
$$


Application of Theorem 7.7.1 of [9] to $\left(I_{\varepsilon}^{\mu}(t)\right)^{\wedge}$ and $\left((\partial / \partial t) I_{\varepsilon}^{\mu}(t)\right)^{\wedge}$ given by (3.11) with $t^{-1} v\left(k, k^{\prime}, t\right)$ as a phase function gives the result. Q.E.D.

For $J$ defined by (3.2) introduce

$$
G_{T}^{\delta}(t)=\int_{T}^{t} \mu(t-s) \sum_{\varepsilon= \pm} J_{\varepsilon}^{\delta}(s) d s
$$

where $0 \leqq t \leqq T, T \geqq 0$, and $\delta=0,1,2,3$. Denote $G^{\delta}=G_{\infty}^{\delta}$. When needed, the dependence of $G^{\delta}$ on $S$ and $\beta$ will be indicated by $G^{\delta}(S, \beta, t)$.

Theorem 3.4. There are constants $C_{n, v}$ such that

$$
|k|^{2+v}|k t|^{n}\left|\left(G_{T_{1}}^{\delta}(t)\right)^{\wedge}(k)-\left(G_{T_{2}}^{\delta}(t)\right)^{\wedge}(k)\right| \leqq C_{n, v} T^{-v}
$$

and

$$
|k|^{1+v}|k t|^{n}\left|\left(\frac{\partial}{\partial t}\left(G_{T_{1}}^{\delta}(t)-G_{T_{2}}^{\delta}(t)\right)^{\wedge}(k)\right)\right| \leqq C_{n, v} T^{-v}
$$

for all $T>0, T_{1} \geqq T, T_{2} \geqq T, t \leqq T, v \geqq 0$ and integers $n \geqq 0$. There are also constants $C_{n}$ such that

$$
|k|^{2}|k t|^{n}\left|\left(G^{\delta}(t)\right)^{\wedge}(k)\right| \leqq C_{n}
$$

and

$$
|k||k t|^{n}\left|\left(\frac{\partial}{\partial t} G^{\delta}(t)\right)^{\hat{(}}(k)\right| \leqq C_{n}
$$

for all $t \geqq 0, n \geqq 0$. The support of $\left(G^{\delta}(t)\right)^{\wedge}$ is contained in a compact set independent of $t \geqq 0$.

Proof. The support property for $\left(G^{\delta}(t)\right)^{\wedge}$ is a direct consequence of that given by Lemma 3.1 for $\left(J_{\varepsilon}^{\delta}(t)\right)^{\wedge}$.

Lemma 3.1 and the definition (3.14) of $G_{T}^{\delta}$ give for $v \geqq 0, n \geqq 0$, and

$$
\begin{aligned}
T & \leqq T_{1} \leqq T_{2}:\left|\left(G_{T_{1}}^{\delta}(t)-G_{T_{2}}^{\delta}(t)\right)^{\wedge}(k)\right| \\
& =\left|\int_{T_{1}}^{T_{2}} \frac{\sin (|k|(t-s))}{|k|} \sum_{\varepsilon= \pm}\left(J_{\varepsilon}^{\delta}(s)\right)^{\wedge}(k) d s\right| \leqq \frac{1}{|k|} \int_{T_{1}}^{T_{2}}(1+|k| s)^{-(n+v+2)} C_{n+v} d s \\
& \leqq \frac{2 C_{n+v}}{n+v+1}\left(|k|^{2}(1+|k| T)^{n+v+1}\right)^{-1} .
\end{aligned}
$$

This gives

$$
\begin{aligned}
& |k|^{2+v}\left|\left(G_{T_{1}}^{\delta}(t)-G_{T_{2}}^{\delta}(t)\right)^{\wedge}(k)\right||k T|^{n} \\
& \quad \leqq \frac{2 C_{n+v}}{n+v+1}(|k| T)^{v}(1+|k T|)^{-1} T^{-v} \leqq \frac{2 C_{n+v}}{n+v+1} T^{-v},
\end{aligned}
$$

which proves the first inequality of the theorem. Hence $G^{\delta}(t)=G_{\infty}^{\delta}(t)$ exists in the sense of (3.15). The second is proved in a similar way. As $G_{t}^{\delta}(t)=0$, the third inequality of the theorem follows from (3.15) by putting $v=0, T_{2}=\infty$, and $T_{1}=T=t$. The last inequality is proved in a similar way. Q.E.D. 
$G$ given by (3.14) has, as a function of $S$ and $\beta$, the following property:

Theorem 3.5. There are finite constants $C_{n}, n \geqq 0$ such that:

i)

$$
\begin{aligned}
& \left.|k|^{2}(1+|k t|)^{n} \mid\left(G^{\delta}\left(S^{(1)}, \beta^{(1)}, t\right)\right)-G^{\delta}\left(S^{(2)}, \beta^{(2)}, t\right)\right)^{\wedge}(k) \mid \\
& \quad \leqq C_{n}\left(\tau_{1}(t)(1+t)^{-1}+\tau_{2}(t)\right),
\end{aligned}
$$

for all $t \geqq 0, k \in R^{3}, n \geqq 0$, and $\delta=0,1,2,3$.

ii)

$$
\begin{aligned}
& \mid\left(\nabla^{n}\left(G^{\delta}\left(S^{(1)}, \beta^{(1)}, t\right)-G^{\delta}\left(S^{(2)}, \beta^{(2)}, t\right)\right)(x) \mid\right. \\
& \quad \leqq C_{n}\left((1+t)^{-(1+n)}\left(\tau_{1}(t)(1+t)^{-1}+\tau_{2}(t)\right)\right.
\end{aligned}
$$

for all $t \geqq 0, x \in R^{3}, n \geqq 0$, and $\delta=0,1,2,3$.

iii)

$$
\begin{aligned}
& \left|\left(\nabla^{n} G^{\delta}(S, \beta, t)\right)\left(x_{1}\right)-\left(\nabla^{n} G^{\delta}(S, \beta, t)\right)\left(x_{2}\right)\right| \\
& \quad \leqq C_{n}(1+t)^{-(2+n)}\left|x_{1}-x_{2}\right|
\end{aligned}
$$

for all $x_{1}, x_{2} \in R^{3}, t \geqq 0$, and $\delta=0,1,2,3$.

Proof. Lemma 3.2 and the definition of $G$ give that the left-hand side of the inequality in i) is smaller than

$$
\begin{aligned}
& |k|^{2}(1+|k t|)^{n} \int_{t}^{\infty}|k|^{-1} C_{n}(1+|k s|)^{-(n+2)}\left(\tau_{1}(s)(1+s)^{-1}+\tau_{2}(s)\right) d s \\
& \quad \leqq C_{n}(1+|k t|)^{n}\left(\tau_{1}(t)(1+t)^{-1}+\tau_{2}(t)\right) \int_{|k| t}^{\infty}(1+s)^{-(n+2)} d s \\
& =C_{n}(1+n)^{-1}\left(\tau_{1}(t)(1+t)^{-1}+\tau_{2}(t)\right)(1+|k t|)^{-1},
\end{aligned}
$$

which proves the statement i) of the theorem. ii) is a direct consequence of i) and iii) is a direct consequence of Theorem 3.4. Q.E.D.

For $I_{\varepsilon}^{\mu}(t)$ defined by formula (3.8) introduce

$$
H_{T}^{\delta}(t)=\int_{T}^{t} \mu(t-s) \sum_{\varepsilon= \pm} I_{\varepsilon}^{\delta}(s) d s,
$$

where $0 \leqq t \leqq T$ and $\delta=0,1,2,3$. Denote $H^{\delta}=H_{\infty}^{\delta}$.

Theorem 3.6. $H_{T}^{\delta}(t)$ converges to $H^{\delta}(t)$ and $(\partial / \partial t) H_{T}^{\delta}(t)$ to $(\partial / \partial t) H^{\delta}(t)$ in $W^{n, 2}\left(R^{3}, C^{4}\right)$ as $T \rightarrow \infty$, for every $t \geqq 0, n=0,1 \ldots$ and $\delta=0,1,2,3$. Moreover

$$
\left\|H^{\delta}(t)\right\|_{W^{n, 2}} \leqq C_{n}(1+t)^{-3 / 2}
$$

and

$$
\left\|\frac{\partial}{\partial t} H^{\delta}(t)\right\|_{W^{n, 2}} \leqq C_{n}(1+t)^{-3 / 2},
$$

for all $t \geqq 0, n \geqq 0, \delta=0,1,2,3$ and some constants $C_{n}<+\infty$.

Proof. It follows from Lemma 3.3 that the support of $H_{T}^{\delta}(t)$ is contained in $K_{1}$ for all $0 \leqq t \leqq T, T \geqq 0$, and $\delta=0,1,2,3$. By Lemma 3.3 and as $\operatorname{supp} H_{T}^{\delta}(t) \subset K_{1}$, there are 
$C_{n}<\infty$ such that

$$
\left\|H_{T_{1}}^{\delta}(t)-H_{T_{2}}^{\delta}(t)\right\|_{W^{n, 2}} \leqq \sum_{\varepsilon} \int_{T_{1}}^{T_{2}}\left\|I_{\varepsilon}^{\delta}(s)\right\|_{W^{n, 2}} d s \leqq C_{n}\left(1+T_{1}\right)^{-1 / 2}
$$

for all $0 \leqq T_{1} \leqq T_{2}, n \geqq 0$, and $\delta=0,1,2,3$, which proves the existence of the limit $H^{\delta}(t)$. Similarly one proves that

$$
(\partial / \partial t) H_{T}^{\delta}(t) \rightarrow(\partial / \partial t) H^{\delta}(t)
$$

By the definition of $H^{\partial}(t)$ :

$$
\begin{aligned}
\left|\left(H^{\delta}(t)\right)^{\wedge}(k)\right| & =\left.\frac{1}{|k|}\right|_{t} ^{\infty} \sin (|k|(t-s)) \sum_{\varepsilon}\left(I_{\varepsilon}^{\delta}(s)\right)^{\wedge}(k) d s \mid \\
& \leqq \frac{1}{2|k|} \sum_{\varepsilon, \varepsilon^{\prime}= \pm}\left|\int_{t}^{\infty} e^{i\left(\varepsilon^{\prime}|k|+2 \varepsilon \omega(k / 2) s\right.}\left(e^{-i 2 \varepsilon \omega(k / 2) s}\left(I_{\varepsilon}^{\delta}(s)\right)^{\wedge}(k)\right) d s\right| .
\end{aligned}
$$

We get after partial integration in $s$, using Lemma 3.3:

$$
\begin{aligned}
\left|\left(H_{\varepsilon}^{\delta}(t)\right)^{\wedge}(k)\right| \leqq & \frac{1}{2|k|} \sum_{\varepsilon, \varepsilon^{\prime}} \mid \frac{e^{i \varepsilon^{\prime}|k|}}{\varepsilon^{\prime}|k|+2 \varepsilon \omega(k / 2)}\left(I_{\varepsilon}^{\delta}(t)\right)^{\wedge}(k) \\
& -\int_{t}^{\infty} \frac{e^{i\left(\varepsilon^{\prime}|k|+2 \varepsilon \omega(k / 2)\right) s}}{\varepsilon^{\prime}|k|+2 \varepsilon \omega(k / 2)} \frac{\partial}{\partial s}\left(e^{-2 i \varepsilon \omega(k / 2)}\left(I_{\varepsilon}^{\delta}(s)\right)^{\wedge}(k)\right) d s \mid \\
\leqq & C|k|^{-1}(2 \omega(k / 2)-|k|)^{-1}(1+t)^{-3 / 2}, \quad t \geqq 0 .
\end{aligned}
$$

As $(2 \omega(k / 2)-|k|)^{-1}$ is uniformly bounded for all $k \in \operatorname{supp}\left(H_{\varepsilon}^{\delta}(t)\right)^{\wedge} \subset K^{\prime}$, where $K^{\prime}$ is independent of $t \geqq 0, \varepsilon= \pm$, and $\delta=0,1,2,3$ it follows from (3.17) that

$$
\left.\sup _{t \geqq 0}(1+t)^{3 / 2}\left\|H^{\delta}(t)\right\|_{W^{n, 2}}\right)<\infty \quad \text { for all } n \geqq 0 .
$$

Similarly one proves the last inequality. Q.E.D.

According to (3.1), (3.14), and (3.16) we have

$$
Q_{T}^{\delta}(t)=G_{T}^{\delta}(t)+H_{T}^{\delta}(t), \quad \text { for } \quad 0 \leqq t \leqq T, \delta=0,1,2,3 .
$$

Corollary 3.7. $\left(Q_{T}(t), \frac{\partial}{\partial t} Q_{T}(t)\right) \in M_{n}$ for all $n \geqq 0, T \geqq 0,0 \leqq t \leqq T$ and has a limit $(Q(t), \dot{Q}(t))$ in $M_{n}$ as $T \rightarrow \infty$. $\dot{Q}$ satisfies $(\partial / \partial t) Q(t)=\dot{Q}(t)$.

Proof. This is a direct consequence of Theorems 3.4 and 3.6. One has only to choose $v>0$ sufficiently small. Q.E.D.

We now turn to the problem of determining the modified asymptotic evolution of the Dirac field.

Introduce

$$
\mathscr{P}_{T}(S, \beta, a, \dot{a}, t)=-i \int_{T}^{t} W_{0}(t, s)\left(\dot{\mu}(s) a_{\delta}+\mu(s) \dot{a}_{\delta}\right) \gamma^{0} \gamma^{\delta} \phi(s) d s,
$$

where $0 \leqq t \leqq T, T \geqq 0, S$ satisfies (2.1), $\beta$ satisfies (2.2) and $\hat{a}_{\delta} \in \mathscr{D}\left(R^{3}, C\right)$, $\hat{\hat{a}}_{\delta} \in \mathscr{D}\left(R^{3}, C\right)$ for $\delta=0,1,2,3$. Denote by $\mathscr{P}=\mathscr{P}_{\infty}$. 
Proposition 3.9. $\left(\mathscr{P}_{T}(S, \beta, a, \dot{a}, t)\right)^{\wedge}$ converges in the topology of $\mathscr{D}\left(R^{3}, C^{4}\right)$, to an element $\left(\mathscr{P}(S, \beta, a, \dot{a}, t)^{\wedge} \in \mathscr{D}\left(R^{3}, C^{4}\right)\right.$. $\mathscr{P}$ has the following decay property:

$$
(1+t)^{n}(\mathscr{P}(S, \beta, a, \dot{a}, t))^{\wedge} \rightarrow 0 \quad \text { in } \quad \mathscr{D}\left(R^{3}, C^{4}\right) \quad \text { as } \quad t \rightarrow \infty
$$

for every $n \geqq 0$.

Proof. It follows from the definition of $\mu, W_{0}, a, \dot{a}$, and $\beta$ that the support of $\left(\left(\dot{\mu}(s) a_{\delta}\right.\right.$ $\left.\left.+\mu(s) \dot{a}_{\delta}\right) \phi(s)\right)^{\wedge}$ is contained in a compact set $K^{\prime}$ independent of $s \geqq 0$ and $\delta=0,1,2,3$ and that it is a sum of expressions

$$
F(k, s)=\int e^{i v\left(k, k^{\prime}, s\right)} u\left(k, k^{\prime}, s\right) d k^{\prime},
$$

where

$$
u\left(k, k^{\prime}, s\right)=f\left(k^{\prime}\right)\left(\beta_{\varepsilon_{2}}(s)\right)^{\wedge}\left(k-k^{\prime}\right), \quad f \in \mathscr{D}\left(R^{3}-\{0\}, C\right), \quad \varepsilon_{2}= \pm
$$

and

$$
v\left(k, k^{\prime}, s\right)=\varepsilon_{1}\left|k^{\prime}\right| s+S_{\varepsilon_{2}}\left(k-k^{\prime}, s\right), \quad \varepsilon_{1}= \pm, \quad k, k^{\prime} \in R^{3}, \quad s \geqq 0 .
$$

Let $\operatorname{supp} f \in K^{\prime \prime}$, where $K^{\prime \prime}$ is a compact subset of $R^{3}-\{0\}$.

For $k^{\prime} \in K^{\prime \prime}$ and for $k-k^{\prime} \in K, v\left(k, k^{\prime}, s\right)$ is $C^{\infty}$ in $k, k^{\prime}$ and there is $v>0$ such that

$$
\left.\left|\varepsilon_{1} k^{\prime}\right| k^{\prime}\right|^{-1}+\varepsilon_{2}\left(k^{\prime}-k\right) \omega\left(k^{\prime}-k\right)^{-1} \mid \geqq 2 v .
$$

By (2.1a) there is then $s_{0} \geqq 0$ such that

$$
s^{-1}\left|\nabla_{k^{\prime}} v\left(k, k^{\prime}, s\right)\right| \geqq v \quad \text { for all } s \geqq s_{0}, k \in K^{\prime}, k-k^{\prime} \in K .
$$

Defining

$$
u_{n}\left(k, k^{\prime}, s\right)=e^{-i v\left(k, k^{\prime}, s\right)} \nabla_{k}^{n} e^{i v\left(k, k^{\prime}, s\right)} u\left(k, k^{\prime}, s\right),
$$

we have

$$
\nabla_{k}^{n} F(k, s)=\int e^{i v\left(k, k^{\prime}, s\right)} u_{n}\left(k, k^{\prime}, s\right) d k^{\prime} .
$$

By (2.1 a) and (2.2a) there is for every $n \geqq 0$ an integer $n^{\prime}(n)$ and finite constants $C_{m, n}$, $m \geqq 0$ such that

$$
\left|\nabla_{k^{\prime}}^{m} u_{n}\left(k, k^{\prime}, s\right)\right| \leqq C_{m, n}(1+t)^{n^{\prime}(n)}
$$

for all $m \geqq 0, k^{\prime} \in K^{\prime \prime}, k \in K^{\prime}$, and $s \geqq 0$. It follows now from Theorem 7.7.1 of [9] that for all $n, m \geqq 0$,

$$
\sup _{\substack{k \in K^{\prime} \\ s \geqq 0}}\left|\nabla_{k}^{n} F(k, s)\right|(1+s)^{m}<\infty
$$

The last inequality shows that

$$
(1+s)^{n}\left(\left(\dot{\mu}(s) a_{\delta}+\mu(s) \dot{a}_{\delta}(s) \phi(s)\right)^{\wedge} \rightarrow 0\right.
$$

in $\mathscr{D}\left(R^{3}, C^{4}\right)$ as $s \rightarrow \infty$. The proposition follows directly from this fact. Q.E.D.

Lemma 3.10. Let $S$ satisfy (2.1 a) and let $\beta$ satisfy (2.2a) and (2.2c). If $\hat{\beta}(k, t)=(\beta(t))^{\wedge}(k)$, then

$$
\sup _{\substack{p, k \in R^{3} \\ t \geqq 0}}\left|\left(\frac{1+t}{1+|p t|^{2}}\right)^{N+1}\left(e^{i S_{\varepsilon}(k-p, t)} \hat{\beta}_{\varepsilon}(k-p, t)-e^{\imath S_{\varepsilon}^{N \cdot 1}(k, t ; p)} \hat{\beta}_{\varepsilon}^{N}(k, t ; p)\right)\right| \leqq C_{N}
$$


for all $N \geqq 0$ and some constants $C_{N}$. Here $S_{\varepsilon}^{N+1}(k, t ; p)$ (respectively $\left.\hat{\beta}_{\varepsilon}^{N}(k, t ; p)\right)$ is the Taylor development of $S_{\varepsilon}(k-p, t)$ (respectively $\hat{\beta}_{\varepsilon}(k-p, t)$ ) to order $N+1$ (respectively $N$ ) in $p$.

Proof. Introduce

$$
\begin{aligned}
f_{N+1}(k, t, p) & =S_{\varepsilon}(k-p, t)-S_{\varepsilon}^{N+1}(k, t ; p), \\
F_{N}(k, t, p) & =\widehat{\beta}_{\varepsilon}(k-p, t)-\widehat{\beta}_{\varepsilon}^{N}(k, t ; p),
\end{aligned}
$$

and

$$
\alpha_{N}^{\varepsilon}(k, t, p)=e^{i S_{\varepsilon}(k-p, t)} \widehat{\beta}_{\varepsilon}(k-p, t)-e^{i S_{\varepsilon}{ }^{+1}(k, t ; p)} \widehat{\beta}_{\varepsilon}(k, t ; p) .
$$

Direct calculation gives:

$$
\begin{aligned}
\left|\alpha_{N}^{\varepsilon}(k, t, p)\right| & =\left|e^{i f_{N+1}(k, t, p)} \hat{\beta}_{\varepsilon}(k-p, t)-\hat{\beta}_{\varepsilon}^{N}(k, t, p)\right| \\
& \leqq\left|e^{i f_{N+1}(k, t, p)} F_{N}(k, t, p)\right|+\left|\left(e^{i f_{N+1}(k, t, p)}-1\right) \hat{\beta}_{\varepsilon}^{N}(k, t, p)\right| \\
& \leqq\left|F_{N}(k, t, p)\right|+\left|f_{N+1}(k, t, p)\right|\left|\hat{\beta}_{\varepsilon}(k, t, p)\right|,
\end{aligned}
$$

where inequality $\left|e^{i x}-1\right| \leqq x, x \in R$ has been used. By hypothesis (2.1a) and (2.2a), $\left|F_{N}(k, t, p)\right| \leqq C_{N}|p|^{N+1}$ and

$$
\left|\mathrm{f}_{N+1}(k, t, p)\right| t^{-1} \leqq C_{N}|p|^{N+2} .
$$

Hence (with new $C_{N}$ )

$$
\left|\alpha_{N}^{\varepsilon}(k, t, p)\right| \leqq C_{N}\left(|p|^{N+1}+|p|^{N+2} t(1+|p|)^{N}\right),
$$

which gives:

$$
\begin{aligned}
& \left|(1+|p t|)^{-2(N+1)} \alpha_{N}^{\varepsilon}(k, t, p)\right| \\
& \quad \leqq C_{N}\left(|p t|^{N+1}+|p t|^{N+2}\left(1+|p|^{N}\right)\right) t^{-(N+1)}(1+|p t|)^{-2(N+1)} \\
& \quad \leqq 2 C_{N} t^{-(N+1)}
\end{aligned}
$$

for $t \geqq 1$. Q.E.D.

Lemma 3.11. Let $G^{\mu}$ be given $b y$ (3.14) and $\alpha_{N} b y$ (3.20). Then there are $C_{N}<\infty$ such that

$$
\left|\int\left(G^{\mu}(t)\right)^{\wedge}\left(k^{\prime}\right) \alpha_{N}^{\varepsilon}\left(k, t, k^{\prime}\right) d k^{\prime}\right| \leqq C_{N}(1+t)^{-(N+2)}
$$

for all $k \in R^{3}, t \geqq 0, \mu=0,1,2,3$, and $N \geqq 0$ and there is a compact set independent of $t$, containing the support of the function

$$
R^{3} \ni k \rightarrow \int\left(G^{\mu}(t)\right)^{\wedge}\left(k^{\prime}\right) \alpha_{N}^{\varepsilon}\left(k, t, k^{\prime}\right) d k^{\prime} .
$$

Proof. For given $N \geqq 0$ and $\mu=0,1,2,3$ denote by

$$
I_{t}^{\prime}(k)=\int\left(G^{\mu}(t)\right)^{\wedge}\left(k^{\prime}\right) \alpha_{N}^{\varepsilon}\left(k, t, k^{\prime}\right) d k^{\prime} .
$$

We have

$$
\begin{aligned}
\left|I_{t}^{\prime}(k)\right|(1+t)^{N+1} \leqq & \int\left(\left|k^{\prime}\right|^{2}\left(1+\left|k^{\prime} t\right|^{2}\right)^{N+2}\left|\left(G^{\mu}(t)\right)^{\wedge}\left(k^{\prime}\right)\right|\right. \\
& \times\left(\frac{1+t}{1+\left|k^{\prime} t\right|^{2}}\right)^{N+1}\left|\alpha_{N}^{\varepsilon}\left(k, t, k^{\prime}\right)\right| \frac{d k^{\prime}}{\left|k^{\prime}\right|^{2}\left(1+\left|k^{\prime} t\right|^{2}\right)} .
\end{aligned}
$$


Majoration of the integrand, with the help of Theorem 3.4 and Lemma 3.10 gives:

$$
\left|I_{t}^{\prime}(k)\right|(1+t)^{N+1} \leqq C_{N} \int \frac{d k^{\prime}}{\left|k^{\prime}\right|^{2}\left(1+\left|k^{\prime} t\right|^{2}\right)}=C_{N^{\prime}} t^{-1} \int_{R^{3}} \frac{d p}{|p|^{2}\left(1+|p|^{2}\right)},
$$

where the last integral is finite. Hence for $t \geqq 1$, there is $C_{N}^{\prime}$ such that $\left|I_{t}^{\prime}(k)\right|$ $\leqq C_{N}^{\prime}(1+t)^{-(N+2)}$.

As $\left|I_{t}^{\prime}(k)\right|$ is uniformly bounded, this proves the lemma. Q.E.D.

Introduce:

$$
\lambda(S, \beta, t)=-i W_{0}(0, t) \gamma^{0} \gamma^{\mu} G_{\mu}(t) \phi(t),
$$

where $t \geqq 0, S$ satisfies (2.1), $\beta$ satisfies (2.2), $W_{0}$ is given by (1.5) and $G$ is given by (3.14). Introduce also $\lambda_{1}, \lambda_{2}, \lambda_{3}$, and $\lambda_{4}$ by:

$$
\begin{aligned}
& \left(\lambda_{1}(S, \beta, t)\right)^{\wedge}(k)=-i \sum_{\varepsilon} P_{\varepsilon}(k) \gamma^{0} \gamma_{\mu} G^{\mu}\left(-\nabla_{k} S_{\varepsilon}(k, t), t\right) P_{\varepsilon}(k)\left(\beta_{\varepsilon}(t)\right)^{\wedge}(k), \\
& \left(\lambda_{2}(S, \beta, t)\right)^{\wedge}(k)=-i \sum_{\varepsilon}\left(P_{\varepsilon}(k) \gamma^{0} \gamma_{\mu} G^{\mu}\left(-\nabla_{k} S_{\varepsilon}(k, t), t\right) P_{-\varepsilon}(k)\left(\beta_{\varepsilon}(t)\right)^{\wedge}(k)\right. \\
& +P_{\varepsilon}(k) \gamma^{0} \gamma_{\mu} \int\left(G^{\mu}(t)\right)^{\wedge}\left(k^{\prime}\right) e^{i\left(\nabla_{k} S_{\varepsilon}(k, t)\right)\left(k^{\prime}\right)} \\
& \left.\left(\left(e^{(i / 2)\left(\nabla_{k}^{2} S_{\varepsilon}(k, t)\right)\left(k^{\prime}\right)}-1\right)\left(\beta_{\varepsilon}(t)\right)^{\wedge}\left(k^{\prime}\right)-\left(\nabla_{k}\left(\beta_{\varepsilon}(t)\right)^{\wedge}(k)\right)\left(k^{\prime}\right)\right)\right) d k^{\prime}, \\
& \quad\left(\lambda_{3}(S, \beta, t)\right)^{\wedge}(k)=-i \sum_{\varepsilon} e^{i\left(S_{\varepsilon}(k, t)-S_{-\varepsilon}(k, t)\right)} P_{\varepsilon}(k) \gamma^{0} \gamma_{\mu} \int\left(\left(G^{\mu}(t)\right)^{\wedge}\left(k^{\prime}\right)\right. \\
& \left.\times e^{i\left(\nabla_{k} S_{\varepsilon}(k, t)\right)\left(k^{\prime}\right)}\left(e^{(i / 2)\left(\nabla_{k}^{2} S_{\varepsilon}(k, t)\right)\left(k^{\prime}\right)}\left(\beta_{\varepsilon}(t)\right)^{\wedge}(k)-\left(\nabla_{k}\left(\beta_{\varepsilon}(t)\right)^{\wedge}(k)\right)\left(k^{\prime}\right)\right)\right) d k^{\prime}, \\
& \quad\left(\lambda_{4}(S, \beta, t)\right)^{\wedge}(k)=-i \sum_{\varepsilon, \varepsilon^{\prime}} e^{i\left(S_{\varepsilon}(k, t)-S_{\varepsilon^{\prime}}(k, t)\right)} P_{\varepsilon^{\prime}}(k) \gamma^{0} \gamma_{\mu} \int\left(\left(G^{\mu}(t)\right)^{\wedge}\left(k^{\prime}\right)\right. \\
& \left.\times e^{-i\left(\nabla_{k} S_{\varepsilon}(k, t)\right)\left(k^{\prime}\right)}\left(e^{(i / 2)\left(\nabla_{k}^{2} S_{\varepsilon}(k, t)\left(k^{\prime}\right)\right.}-1\right)\left(-\nabla_{k}\left(\beta_{\varepsilon}(t)\right)^{\wedge}(k)\right)\left(k^{\prime}\right)\right) d k^{\prime},
\end{aligned}
$$

where $\left(\nabla_{k} f(k)\right)\left(k^{\prime}\right)$ is the Fréchet derivative of $f$ with respect to $k$ in the direction $k^{\prime}$ and $G_{\mu}(x, t)=\left(G^{\mu}(t)\right)(x)$.

Theorem 3.12. For every $n \geqq 0$ there is $C_{n}<+\infty$ such that

$$
\left\|(1-\Delta)^{n / 2}\left(\lambda(S, \beta, t)-\lambda_{1}(S, \beta, t)-\lambda_{2}(S, \beta, t)-\lambda_{3}(S, \beta, t)\right)\right\|_{L^{2}} \leqq C_{n}(1+t)^{-3}
$$

for all $t \geqq 0$.

Proof. By construction

$$
\left(W_{0}(t, 0)\left(\lambda(S, \beta, t)-\sum_{i=1}^{4} \lambda_{i}(S, \beta, t)\right) \hat{)}(k)=-i \gamma^{0} \gamma_{\mu} \int\left(G^{\mu}(t)\right)^{\wedge}\left(k^{\prime}\right) \sum_{\varepsilon} \alpha_{1}^{\varepsilon}\left(k, t, k^{\prime}\right) d k^{\prime},\right.
$$

where $\alpha_{1}^{\varepsilon}$ is given by (3.20). Lemma (3.11), with $N=1$, shows then the existence of constants $C_{n}$ such that

$$
\left\|\lambda(S, \beta, t)-\sum_{i=1}^{5} \lambda_{i}(S, \beta, t)\right\|_{W^{n, 2}} \leqq C_{n}(1+t)^{-3},
$$


for all $t \geqq 0$ and $n \geqq 0$. Hence the theorem follows from the inequality

$$
\left\|\lambda_{4}(S, \beta, t)\right\|_{W^{n, 2}} \leqq C_{n}(1+t)^{-3}, \quad n \geqq 0, t \geqq 0,
$$

which we now prove.

By (3.25) and by $\left|e^{i x}-1\right| \leqq x, x \in R$ there is a constant $C$ such that

$$
\left|\left(\lambda_{4}(S, \beta, t)\right)^{\wedge}(k)\right| \leqq C \sum_{\mu, \varepsilon} \int\left|\left(G^{\mu}(t)\right)^{\wedge}\left(k^{\prime}\right)\right|\left|\left(\nabla_{k}^{2} S_{\varepsilon}(k, t)\right)\left(k^{\prime}\right)\right|\left|\left(\nabla_{k}\left(\beta_{\varepsilon}(t)\right)^{\wedge}(k)\right)\left(k^{\prime}\right)\right| d k^{\prime} .
$$

Formulas (2.1) and (2.2) give that

$$
\left|\nabla_{k}^{2} S_{\varepsilon}(k, t)\right| \leqq C^{\prime}(t+1) \text { and }\left|\nabla_{k}\left(\beta_{\varepsilon}(t)\right)^{\wedge}(k)\right| \leqq C^{\prime}
$$

for all $k \in R^{3}, t \geqq 0$ and some $C^{\prime}<+\infty$. This introduced into the last inequality for $\lambda_{4}$, gives together with Theorem 3.4:

$$
\left|\left(\lambda_{4}(S, \beta, t)\right)^{\wedge}(k)\right| \leqq C^{\prime \prime} \sum_{\mu} \int\left|\left(G^{\mu}(t)\right)^{\wedge}\left(k^{\prime}\right)\right|\left|k^{\prime}\right|^{3}(1+t) d k^{\prime} \leqq C^{\prime \prime \prime}(1+t)^{-3}
$$

for all $k \in R^{3}, t \geqq 0$. This inequality and the fact that $\operatorname{supp}\left(\beta_{\varepsilon}(t)\right)^{\wedge} \subset K$, for all $t \geqq 0$, $\varepsilon= \pm$, prove (3.26). Q.E.D.

The following lemma will be useful to show that the function $S_{\varepsilon}, \varepsilon= \pm$, should be chosen close to solutions of the Hamilton-Jacobi equation for an electron in an electromagnetic potential.

Lemma 3.13. Let $f_{\mu} \in C$ for $\mu=0,1,2,3$. Then

$$
\begin{gathered}
P_{\varepsilon}(k) \gamma^{0} \gamma^{\mu} f_{\mu} P_{\varepsilon}(k)=\left(-\varepsilon \sum_{i=1}^{3} k_{i} f_{i}(\omega(k))^{-1}+f_{0}\right) P_{\varepsilon}(k), \\
\left(\lambda_{1}(S, \beta, t)\right)^{\wedge}=i \sum_{\varepsilon= \pm}\left(L_{\varepsilon}(S, \beta, t)-\varepsilon \omega\right) P_{\varepsilon}\left(\beta_{\varepsilon}(t)\right)^{\wedge},
\end{gathered}
$$

where

$$
\begin{aligned}
\left(L_{\varepsilon}(S, \beta, t)\right)(k)= & \varepsilon \omega(k)+\sum_{i=1}^{3} \varepsilon(\omega(k))^{-1} k_{i} G_{i}\left(-\nabla_{k} S_{\varepsilon}(k, t), t\right) \\
& -G_{0}\left(-\nabla_{k} S_{\varepsilon}(k, t), t\right), \quad \varepsilon= \pm .
\end{aligned}
$$

Proof. Direct calculation, using that $\gamma_{\mu} \gamma_{\nu}+\gamma_{\nu} \gamma_{\mu}=2 g_{\mu \nu}$ gives:

$$
\sum_{j=1}^{3} \gamma^{0} \gamma^{j} P_{\varepsilon}(k) f_{j}=\sum_{j=1}^{3}\left(P_{-\varepsilon}(k) \gamma^{0} \gamma^{j} f_{j}-\varepsilon(\omega(k))^{-1} k_{j} f_{j} P_{\varepsilon}(k)\right) .
$$

Applying $P_{\varepsilon}(k)$ from the left gives

$$
\sum_{j=1}^{3} P_{\varepsilon}(k) \gamma^{0} \gamma^{j} f_{j} P_{\varepsilon}(k)=-\varepsilon(\omega(k))^{-1} \sum_{j=1}^{3} k_{j} f_{j} P_{\varepsilon}(k),
$$

which proves the first equality of the lemma. The second equality follows from the first equality, formula (3.22) and from the fact that $W_{0}(t, s)$ commutes with $P_{\varepsilon}(\partial)$. Q.E.D.

Remark 3.14. We note that in expression (3.27)

$$
\left(L_{\varepsilon}(S, \beta, t)\right)(k)=\sum_{i=1}^{3} \varepsilon(\omega(k))^{-1} k_{i} G_{i}\left(-\nabla_{k} S_{\varepsilon}(k, t), t\right)-G_{0}\left(-\nabla_{k} S_{\varepsilon}(k, t), t\right)+\varepsilon \omega(k)
$$


is the sum of the zeroth and the first order terms in the Taylor development of

$$
\varepsilon \omega\left(k+\vec{G}\left(-\nabla_{k} S_{\varepsilon}(k, t), t\right)\right)-G_{0}\left(-\nabla_{k} S_{\varepsilon}(k, t), t\right)
$$

with respect to $G$. Here $\vec{G}=\left(G_{1}, G_{2}, G_{3}\right)$.

Lemma 3.15. In the situation of Lemma 3.2 one has:

i)

$\left|\nabla_{k}^{n}\left(\left(L_{\varepsilon}\left(S^{(1)}, \beta^{(1)}, t\right)\right)(k)-\left(L_{\varepsilon}\left(S^{(2)}, \beta^{(2)}, t\right)\right)(k)\right)\right|$

$$
\leqq C_{n}\left((1+t)^{-2} \tau_{1}(t)+(1+t)^{-1} \tau_{2}(t)\right)
$$

ii)

$$
\left|\nabla_{k}^{n}\left(\left(L_{\varepsilon}(S, \beta, t)\right)(k)-\varepsilon \omega(k)\right)\right| \leqq C_{n}(1+t)^{-1},
$$

iii)

$$
\left|\nabla_{k}^{n} \frac{\partial}{\partial t}\left(L_{\varepsilon}(S, \beta, t)\right)(k)\right| \leqq C_{n}(1+t)^{-2},
$$

iv)

v)

$$
\begin{gathered}
\left.\mid \nabla_{k}^{n}\left(\lambda_{1}(S, \beta, t)\right)^{\wedge}(k)\right) \mid \leqq C_{n}(1+t)^{-1}, \\
\left|\nabla_{k}^{n}\left(\lambda_{1}\left(S^{(1)}, \beta^{(1)}, t\right)-\lambda_{1}\left(S^{(2)}, \beta^{(2)}, t\right)\right)^{\wedge}(k)\right|
\end{gathered}
$$$$
\leqq C_{n}\left((1+t)^{-2} \tau_{1}(t)+(1+t)^{-1} \tau_{2}(t)\right),
$$

for all $t \geqq 0, k \in R^{3}, n \geqq 0, \varepsilon= \pm$ and some constants $C_{n}<\infty$.

Proof. We prove the lemma for $n=0$. For $n>0$ the lemma can be proved by induction.

Let $0 \leqq \mu \leqq 3$. Theorem 3.5 gives

$$
\begin{aligned}
&\left|G_{\mu}^{(1)}\left(-\nabla_{k} S_{\varepsilon}^{(1)}(k, t), t\right)-G_{\mu}^{(2)}\left(-\nabla_{k} S_{\varepsilon}^{(2)}(k, t), t\right)\right| \\
& \leqq\left|G_{\mu}^{(1)}\left(-\nabla_{k} S_{\varepsilon}^{(1)}(k, t), t\right)-G_{\mu}^{(1)}\left(-\nabla_{k} S_{\varepsilon}^{(2)}(k, t), t\right)\right| \\
&+\left|G_{\mu}^{(1)}\left(-\nabla_{k} S_{\varepsilon}^{(2)}(k, t), t\right)-G_{\mu}^{(2)}\left(-\nabla_{k} S_{\varepsilon}^{(2)}(k, t), t\right)\right| \\
& \leqq C\left((1+t)^{-2} \tau_{1}(t)+(1+t)^{-1}\left((1+t)^{-1} \tau_{1}(t)+\tau_{2}(t)\right)\right) \\
& \leqq C^{\prime}\left((1+t)^{-2} \tau_{1}(t)+(1+t)^{-1} \tau_{2}(t)\right) .
\end{aligned}
$$

Inequality i) with $n=0$, follows directly from (3.29). Inequality v) with $n=0$, follows from (3.29) and

$$
\begin{aligned}
& \left|\left(\lambda_{1}\left(S^{(1)}, \beta^{(1)}, t\right)-\lambda_{1}\left(S^{(2)}, \beta^{(2)}, t\right)\right)^{\wedge}(k)\right| \\
& \quad \leqq \\
& \quad C \sum_{\varepsilon, \mu}\left(\left|G_{\mu}^{(1)}\left(-\nabla_{k} S_{\varepsilon}^{(1)}(k, t), t\right)\right|\left|\left(\beta_{\varepsilon}^{(1)}(t)-\beta_{\varepsilon}^{(2)}(t)\right)^{\wedge}(k)\right|\right. \\
& \left.\quad+\left|G_{\mu}^{(1)}\left(-\nabla_{k} S_{\varepsilon}^{(1)}(k, t), t\right)-G_{\mu}^{(2)}\left(-\nabla_{k} S_{\varepsilon}^{(2)}(k, t), t\right)\right|\left|\left(\beta_{\varepsilon}^{(2)}(t)\right)^{\wedge}(k)\right|\right) \\
& \leqq \\
& \quad C^{\prime}\left((1+t)^{-1} \tau_{2}(t)+(1+t)^{-2} \tau_{1}(t)+(1+t)^{-1} \tau_{2}(t)\right) .
\end{aligned}
$$

Inequality ii) (respectively iv)) is obtained from inequality i) (respectively v)) by putting $\beta=\beta^{(1)}, S=S^{(1)}$, and $\beta^{(2)}=0$ (which implies that $\tau_{2}=1$ ). We omit the proof of iii). Q.E.D.

Lemma 3.16. Let $S$ and $\beta$ satisfy (2.1) and (2.2) and let $S^{(1)}, \beta^{(1)}, S^{(2)}, \beta^{(2)}$ be as in Lemma 3.2. Then

$$
\begin{gathered}
\quad \nabla_{k}^{n}\left(\lambda_{2}(S, \beta, t)\right)^{\wedge}(k) \mid \leqq C_{n}(1+t)^{-2}, \\
\left|\nabla_{k}^{n}\left(\lambda_{2}\left(S^{(1)}, \beta^{(1)}, t\right)-\lambda_{2}\left(S^{(2)}, \beta^{(2)}, t\right)\right)^{\wedge}(k)\right| \\
\leqq \\
\quad C_{n}(1+t)^{-2}\left((1+t)^{-1} \tau_{1}(t)+\tau_{2}(t)\right),
\end{gathered}
$$$$
\text { ii) }
$$

for all $t \geqq 0, n \geqq 0, k \in R^{3}$ and some $C_{n}$. 
Proof. We prove the lemma for $n=0$. The case $n \geqq 1$ can be proved by induction.

The definition, of $\lambda_{2}$ [formula (3.23)] gives:

$$
\left|\left(\lambda_{2}\left(S^{(1)}, \beta^{(1)}, t\right)-\lambda_{2}\left(S^{(2)}, \beta^{(2)}, t\right)\right)^{\wedge}(k)\right| \leqq \int\left|f^{(1)}\left(k, k^{\prime}, t\right)-f^{(2)}\left(k, k^{\prime}, t\right)\right| d k^{\prime},
$$

where

$$
\begin{aligned}
& f^{(j)}\left(k, k^{\prime}, t\right)=\sum_{\varepsilon}\left(G_{\mu}^{(j)}(t)\right)^{\wedge}\left(k^{\prime}\right) e^{-i\left(\nabla_{k} S_{\varepsilon}^{(j)}(k, t)\right)\left(k^{\prime}\right)} \\
& \quad \times\left(\left(e^{(i / 2)\left(\nabla_{k}^{2} S_{\varepsilon}^{(j)}(k, t)\right)\left(k^{\prime}\right)}-1\right)\left(\beta_{\varepsilon}^{(j)}(t)\right)^{\wedge}(k)-\left(\nabla_{k}\left(\beta_{\varepsilon}^{(j)}(t)\right)^{\wedge}(k)\right)\left(k^{\prime}\right)\right) .
\end{aligned}
$$

Let $a_{1}, \ldots, a_{m}$ and $b_{1}, \ldots, b_{m}$ be complex numbers. Then

$$
\left|a_{1} a_{2} \ldots a_{m}-b_{1} b_{2} \ldots b_{m}\right| \leqq \sum_{i=1}^{m}\left|a_{i}-b_{i}\right| \prod_{j \neq i}\left(\left|a_{j}\right|+\left|b_{j}\right|\right) .
$$

Application of this inequality on $\left|f^{(1)}-f^{(2)}\right|$ gives:

$$
\begin{aligned}
& \left|f^{(1)}\left(k, k^{\prime}, t\right)-f^{(2)}\left(k, k^{\prime}, t\right)\right| \\
& \quad \leqq \\
& \quad \sum_{\varepsilon, \mu}\left(\left|\left(G_{\mu}^{(1)}(t)-G_{\mu}^{(2)}(t)\right)^{\wedge}\left(k^{\prime}\right)\right|\left(\left|k^{\prime}\right|^{2} t+\left|k^{\prime}\right|\right)\right. \\
& \quad+\left(\left|\left(G_{\mu}^{(1)}(t)\right)^{\wedge}\left(k^{\prime}\right)\right|+\left|\left(G_{\mu}^{(2)}(t)\right)^{\wedge}\left(k^{\prime}\right)\right|\right)\left(\left|\nabla_{k}^{2}\left(S_{\varepsilon}^{(1)}(k, t)-S_{\varepsilon}^{(2)}(k, t)\right)\right|\left|k^{\prime}\right|^{2}\right. \\
& \left.\left.\quad+\left|k^{\prime}\right|^{2} t\left|\left(\beta_{\varepsilon}^{(1)}(t)-\beta_{\varepsilon}^{(2)}(t)\right)^{\wedge}\left(k^{\prime}\right)\right|+\left|\nabla_{k}\left(\beta_{\varepsilon}^{(1)}(t)-\beta_{\varepsilon}^{(2)}(t)\right)^{\wedge}(k)\right|\left|k^{\prime}\right|\right)\right),
\end{aligned}
$$

where we have used that $(1+t)^{-1} S_{\varepsilon}^{(i)}(k, t)$ and $\left(\beta_{\varepsilon}^{(i)}(\mathrm{t})\right)^{\wedge}(k)$ are bounded together with their derivatives in $k$. The norms of expressions involving derivatives are the norms of multilinear maps. Using Theorems 3.5 i), 3.4 and the hypothesis of Lemma 3.2 we get:

$$
\begin{aligned}
& \left|f^{(1)}\left(k, k^{\prime}, t\right)-f^{(2)}\left(k, k^{\prime}, t\right)\right| \\
& \quad \leqq C^{\prime}\left|k^{\prime}\right|^{2}\left(1+\left|k^{\prime}\right| t\right)^{-4}\left(\left((1+t)^{-1} \tau_{1}(t)+\tau_{2}(t)\right)\left(\left|k^{\prime}\right|^{2} t+\left|k^{\prime}\right|\right)\right. \\
& \left.\quad+\tau_{1}(t)\left|k^{\prime}\right|^{2}+\left|k^{\prime}\right|^{2} t \tau_{2}(t)+\tau_{2}(t)\left|k^{\prime}\right|\right) .
\end{aligned}
$$

Integration of the last inequality in $k^{\prime}$ gives:

$$
\begin{aligned}
& \int\left|f^{(1)}\left(k, k^{\prime}, t\right)-f^{(2)}\left(k, k^{\prime}, t\right)\right| d k^{\prime} \\
& \leqq \\
& \leqq
\end{aligned}
$$

Introduce

$$
\left(l_{\varepsilon}(S, \beta, t)\right)^{\wedge}(k)=-\varepsilon(2 i \omega(k))^{-1} e^{-i S_{\varepsilon}(k, t)} P_{-\varepsilon}(k)\left(W_{0}(t, 0) \lambda_{3}(S, \beta, t)\right)^{\wedge}(k) .
$$

Lemma 3.17. Let $S$ and $\beta$ satisfy (2.1) and (2.2) and let $S^{(1)}, \beta^{(1)}, S^{(2)}, \beta^{(2)}$ be as in Lemma 3.2. Then

$$
\begin{gathered}
\left|\nabla_{k}^{n}\left(l_{\varepsilon}(S, \beta, t)\right)^{\wedge}(k)\right| \leqq C_{n}(1+t)^{-1}, \\
\left|\nabla_{k}^{n} \frac{\partial}{\partial t}\left(l_{\varepsilon}(S, \beta, t)\right)^{\wedge}(k)\right| \leqq C_{n}(1+t)^{-2},
\end{gathered}
$$


iii)

$$
\begin{aligned}
& \left|\nabla_{k}^{n}\left(l_{\varepsilon}\left(S^{(1)}, \beta^{(1)}, t\right)-l_{\varepsilon}\left(S^{(2)}, \beta^{(2)}, t\right)\right)^{\wedge}(k)\right| \\
& \quad \leqq C_{n}(1+t)^{-1}\left((1+t)^{-1} \tau_{1}(t)+\tau_{2}(t)\right), \\
& \left|\nabla_{k}^{n} \frac{\partial}{\partial t}\left(l_{\varepsilon}\left(S^{(1)}, \beta^{(1)}, t\right)-l_{\varepsilon}\left(S^{(2)}, \beta^{(2)}, t\right)\right)^{\wedge}(k)\right| \\
& \quad \leqq C_{n}(1+t)^{-2}\left((1+t)^{-1} \tau_{1}(t)+\tau_{2}(t)\right),
\end{aligned}
$$

iv)

for all $t \geqq 0, n \geqq 0, \varepsilon= \pm, k \in \mathrm{R}^{3}$ and some $C_{n}<+\infty$.

The proof of this lemma is so similar to that of Lemma 3.16, that we omit it.

Let $T=\gamma^{1} \gamma^{2} \gamma^{3}$. Then $T=T^{1}$ and $P_{+}(k)=T P_{-}(k) T$. We get from (3.30):

$$
\sum_{\varepsilon= \pm} e^{i S_{\varepsilon}(k, t)}\left(l_{\varepsilon}(S, \beta, t)\right)^{\wedge}(k)=\left(T W_{0}(t, 0) T l(S, \beta, t)\right)^{\wedge}(k) .
$$

Proposition 3.18. There are $C_{n}<+\infty$ such that

$$
\left\|\int_{\infty}^{t} W_{0}(s, 0) \lambda_{3}(S, \beta, s) d s-T W_{0}(t, 0) T l(S, \beta, t)\right\|_{W^{n, 2}} \leqq C_{n}(1+t)^{-2}
$$

for all $t \geqq 0$ and $n \geqq 0$.

The proof, which we omit, is a direct consequence of Lemma 3.17 and the definition of $\lambda_{3}$.

We can now determine $S$ and $\beta$. Let

$$
S_{\varepsilon}^{(0)}(k, 0)=\varepsilon \omega(k) t, \quad\left(S^{(0)}(t)\right)(k)=S^{0}(k, t), \quad \beta^{(0)}=\alpha .
$$

We define recursively:

$$
\begin{gathered}
S^{(1)}(t)=\int_{0}^{t} L\left(S^{(0)}, \beta^{(0)}, s\right) d s, \\
S^{(n+1)}(t)=S^{(n)}(t)+\int_{\infty}^{t}\left(L\left(S^{(n)}, \beta^{(n)}, s\right)-\frac{\partial}{\partial s} S^{(n)}(s)\right) d s, \quad n \geqq 1
\end{gathered}
$$

and

$$
\beta_{\varepsilon}^{(n+1)}(t)=\beta_{\varepsilon}^{(0)}+P_{\varepsilon}(\partial) \int_{\infty}^{t} \lambda_{2}\left(S^{(n)}, \beta^{(n)}, s\right) d s+l_{\varepsilon}\left(S^{(n)}, \beta^{(n)}, t\right), \quad n \geqq 0 .
$$

$S$ (respectively $\beta$ ) will later be defined to be equal to $S^{(n)}$ (respectively $\beta^{(n)}$ ) for some $n$, sufficiently large.

Lemma 3.19. Let

$$
\begin{aligned}
\tau_{1}^{(n)}(t) & =(1+t)^{-(n-1)}(\log (1+t)+1), \quad n \geqq 2, \\
\tau_{1}^{(1)}(t) & =\log (1+t),
\end{aligned}
$$

and let

$$
\begin{aligned}
\tau_{2}^{(n)}(t) & =(1+t)^{-n}(\log (1+t)+1), \quad n \geqq 2, \\
\tau_{2}^{(1)}(t) & =(1+t)^{-1} .
\end{aligned}
$$

There are then constants $C_{m, n}$ such that

$$
\left|\nabla_{k}^{m} \frac{\partial}{\partial t}\left(S_{\varepsilon}^{(n)}(k, t)-S_{\varepsilon}^{(n-1)}(k, t)\right)\right| \leqq C_{m, n}\left|\frac{d}{d t} \tau_{1}^{(n)}(t)\right|
$$


and

$$
\left|\nabla_{k}^{m} \frac{\partial}{\partial t}\left(\beta_{\varepsilon}^{(n)}(t)-\beta_{\varepsilon}^{(n-1)}(t)\right)^{\wedge}(k)\right| \leqq C_{m, n}\left|\frac{d}{d t} \tau_{2}^{(n)}(t)\right|
$$

for all $k \in R^{3}, t \geqq 0, m \geqq 0$, and $n \geqq 1$. Moreover

$$
\left|\nabla_{k}^{m}\left(S_{\varepsilon}^{(n)}(k, t)-S_{\varepsilon}^{(n-1)}(k, t)\right)\right| \leqq C_{m, n} \tau_{1}^{(n)}(t)
$$

and

$$
\left|\nabla_{k}^{m}\left(\beta_{\varepsilon}^{(n)}(t)-\beta_{\varepsilon}^{(n-1)}(t)\right)(k)\right| \leqq C_{m, n} \tau_{2}^{(n)}(t) .
$$

Proof. By definition

$$
\frac{\partial}{\partial \mathrm{t}}\left(S_{\varepsilon}^{(1)}(t)-S_{\varepsilon}^{(0)}(t)\right)=L\left(S^{(0)}, \beta^{(0)}, t\right)-\varepsilon \omega .
$$

By Lemma 3.15 ii) we then have

$$
\left|\nabla_{k}^{m} \frac{\partial}{\partial t}\left(S_{\varepsilon}^{(1)}(k, t)-S_{\varepsilon}^{(0)}(k, t)\right)\right| \leqq C_{m}(1+t)^{-1}, \quad m \geqq 0, t \geqq 0 .
$$

By definition

$$
\frac{\partial}{\partial t} \beta^{(1)}(t)=\lambda_{2}\left(S^{(0)}, \beta^{(0)}, t\right),
$$

which together with Lemma 3.16i) gives that

$$
\mid \nabla_{k}^{m}\left(\frac{\partial}{\partial t} \beta_{\varepsilon}^{(1)}(t) \hat{)}(k) \mid \leqq C_{m}(1+t)^{-2}, \quad m \geqq 0, t \geqq 0 .\right.
$$

Hence (3.36) and (3.27) are true for $n=1$. Suppose (3.36) and (3.27) true for $n$. By definition

$$
\frac{\partial}{\partial t}\left(S_{\varepsilon}^{(n+1)}(t)-S_{\varepsilon}^{(n)}(t)\right)=L_{\varepsilon}\left(S^{(n)}, \beta^{(n)}, t\right)-L_{\varepsilon}\left(S^{(n-1)}, \beta^{(n-1)}, t\right)
$$

and

$$
\begin{aligned}
\frac{\partial}{\partial t}\left(\beta^{(n+1)}(t)\right. & \left.-\beta^{(n)}(t)\right)=\lambda_{2}\left(S^{(n)}, \beta^{(n)}, t\right)-\lambda_{2}\left(S^{(n-1)}, \beta^{(n-1)}, t\right) \\
& +\frac{\partial}{\partial t}\left(l_{\varepsilon}\left(S^{(n)}, \beta^{(n)}, t\right)-l_{\varepsilon}\left(S^{(n-1)}, \beta^{(n-1)}, t\right) .\right.
\end{aligned}
$$

Lemmas $3.15 \mathrm{i}), 3.16 \mathrm{ii}$ ), and $3.17 \mathrm{iv)}$ now give that

$$
\left|\nabla_{k}^{m} \frac{\partial}{\partial t}\left(S_{\varepsilon}^{(n+1)}(k, t)-S_{\varepsilon}^{(n)}(k, t)\right)\right| \leqq C_{m, n}^{\prime}\left((1+t)^{-2} \tau_{1}^{(n)}(t)+(1+t)^{-1} \tau_{2}^{(n)}(t)\right)
$$

and that

$$
\mid \nabla_{k}^{m} \frac{\partial}{\partial t}\left(\beta^{(n+1)}(t)-\beta^{(n)}(t)^{\wedge}(k) \mid \leqq C_{m, n}^{\prime}\left((1+t)^{-3} \tau_{1}^{(n)}(t)+(1+t)^{-2} \tau_{2}^{(n)}(t)\right)\right.
$$

for some constants $C_{m, n}^{\prime}$. 
The last two inequalities and the inequalities

$$
\begin{array}{ll}
(1+t)^{-2} \tau_{1}^{(n)}(t)+(1+t)^{-1} \tau_{2}^{(n)}(t) \leqq C_{n}\left|\frac{d}{d t} \tau_{1}^{(n+1)}(t)\right|, & n \geqq 1, \\
(1+t)^{-3} \tau_{1}^{(n)}(t)+(1+t)^{-2} \tau_{2}^{(n)}(t) \leqq C_{n}\left|\frac{d}{d t} \tau_{2}^{(n+1)}(t)\right|, & n \geqq 1
\end{array}
$$

show that (3.36a) and (3.37a) are true for $n+1$. Integration of (3.38) and (3.39) gives (3.37) for $n+1$, which by induction proves the lemma. Q.E.D.

We now define

$$
S=S^{(3)}, \quad \beta=\beta^{(3)} .
$$

Remark 3.20. $S$ and $\beta$, defined by (3.40) satisfy the hypotheses (2.1) and (2.2) made on $S$ and $\beta$. In fact (2.1a), (2.1b), (2.2a), and (2.2b) are direct consequences of Lemma 3.19. The support property (2.1c) follows from the fact that $\left(\lambda_{2}\left(S^{(n)}, \beta^{(n)}, t\right)\right)$ has, for each $n$, support in a compact independent of $t \geqq 0$.

Remark 3.21. Instead of defining $S$ by (3.40) as an approximate solution of the equation $(\partial / \partial t) S(t)=L(S, \beta, t)$, we could have defined $S$ to be a solution of the Hamiltonian-Jacobi equation

$$
\left(\frac{\partial}{\partial t} \mathbf{S}_{\boldsymbol{\varepsilon}}(k, t)+G_{0}\left(-\nabla_{k} \mathbf{S}_{\boldsymbol{\varepsilon}}(k, t), t\right)\right)^{2}-\left|k+\vec{G}\left(-\nabla_{k} \mathbf{S}_{\varepsilon}(k, t), t\right)\right|^{2}=m^{2} .
$$

The function $\lambda_{2}$, as defined by formula (3.34), should then be slightly modified. However for simplicity, we have chosen not to study the existence problem for solutions of (3.41).

$S$ and $\beta$ given by (3.40) have been constructed such that the difference

$$
\left(W_{0}(t, 0) \alpha+\int_{\infty}^{t} W_{0}(t, s)\left(\mathscr{D}-i \gamma^{0} \gamma^{\mu} G_{\mu}(s)-\mathscr{L}(s)\right) \phi(s) d s\right)-\phi(t)
$$

is small. This is made precise by the following theorem:

Theorem 3.22. Let $S$ and $\beta$ be given by (3.40), $\phi$ by (2.3), $\mathscr{L}$ by (1.8) and $W_{0}$ by (1.5). Then

i) The strong improper Riemann integral

$$
\int_{\infty}^{t} W_{0}(t, s)\left(\mathscr{D}-i \gamma^{0} \gamma^{\mu} G_{\mu}(s)-\mathscr{L}(s)\right) \phi(s) d s, \quad t \geqq 0
$$

exists in $W^{n, 2}$ for all $n \geqq 0$.

ii) For every $n \geqq 0$ there is $C_{n}<+\infty$ such that

$$
\| W_{0}(t, 0) \alpha+\int_{\infty}^{t} W_{0}(t, s)\left(\mathscr{D}-i \gamma^{0} \gamma^{\mu} G_{\mu}(s)-\mathscr{L}(s) \phi(s) d s-\phi(t) \|_{W^{n, 2}} \leqq C_{n}(1+t)^{-2}\right.
$$

for all $n \geqq 0$.

Proof. Introduce $F_{1}(s)=W_{0}(0, s)\left(\mathscr{D}-i \gamma^{0} \gamma^{\mu} G_{\mu}(s)-\mathscr{L}(s)\right) \phi(s)$ and

$$
F_{2}(s)=W_{0}(0, s)(\mathscr{D}-\mathscr{L}(s)) \phi(s)+\lambda_{1}(s)+\lambda_{2}(s)+\lambda_{3}(s) .
$$


According to Theorem 3.12, $\left\|F_{1}(s)-F_{2}(s)\right\|_{W^{n, 2}} \leqq C_{n}(1+t)^{-3}$ for every $n \geqq 0$, $t \geqq 0$ and some constants $C_{n}<\infty$. For $s \geqq 0$ introduce $F_{3}$ by

$$
\left(F_{3}(s)\right)^{\wedge}(k)=\left(F_{2}(s)\right)^{\wedge}(k)-\left(\sum_{\varepsilon} i\left(\varepsilon \omega(k)-\frac{\partial S_{\varepsilon}(k, s)}{\partial s}\right) P_{\varepsilon}(k)\left(\beta_{\varepsilon}(s)\right)^{\wedge}(k)+\left(\lambda_{1}(s)\right)^{\wedge}(k)\right) .
$$

Lemma 3.13 ii) gives that

$$
\left(F_{2}(s)-F_{3}(s)\right)^{\wedge}(k)=\sum_{\varepsilon} i\left(\left(L_{\varepsilon}(S, \beta, s)\right)(k)-\frac{\partial S_{\varepsilon}(k, s)}{\partial s}\right) P_{\varepsilon}(k)\left(\beta_{\varepsilon}(s)\right)^{\wedge}(k),
$$

which in turn gives, denoting $L_{\varepsilon}(S, \beta, s)$ by $L_{\varepsilon}(s)$,

$$
\left.\left|\left(F_{2}(s)-F_{3}(s)\right)^{\wedge}(k)\right| \leqq C \sum_{\varepsilon}\left|L_{\varepsilon}(s)(k)-\frac{\partial S_{\varepsilon}(k, s)}{\partial s}\right|\left(\mid \beta_{+}(s)\right)^{\wedge}(k)|+|\left(\beta_{-}(s)\right)^{\wedge}(k) \mid\right) .
$$

By the definitions (3.33a) and (3.40) one has

$$
\left(L_{\varepsilon}(S, \beta, s)\right)(k)-\frac{\partial S_{\varepsilon}(k, s)}{\partial s}=\frac{\partial S_{\varepsilon}^{(4)}(k, s)}{\partial s}-\frac{\partial S_{\varepsilon}^{(3)}(k, s)}{{ }^{\prime} s},
$$

which by Lemma 3.19 gives:

$$
\left|\left(L_{\varepsilon}(S, \beta, s)\right)(k)-\frac{\partial S_{\varepsilon}(k, s)}{\partial s}\right| \leqq C(1+s)^{-4}(1+\log (1+s)) .
$$

The two last inequalities and (2.2) give

$$
\left\|F_{2}(s)-F_{3}(s)\right\|_{W^{n, 2}} \leqq C_{n}(1+s)^{-4}(1+\log (1+s)), \quad s \geqq 0,
$$

for every $n \geqq 0$.

The function $F_{3}$ can be written

$$
\begin{aligned}
& \left(F_{3}(s)\right)^{\wedge}(k)=\left(\lambda_{2}(s)+\lambda_{3}(s)\right)^{\wedge}(k) \\
& \quad+\sum_{\varepsilon} i\left(\varepsilon \omega(k)-\frac{\partial S_{\varepsilon}(k, s)}{\partial s}\right) P_{\varepsilon}(k) e^{-i S_{\varepsilon}(k, s)}\left(\phi_{-\varepsilon}(s)\right)^{\wedge}(k) .
\end{aligned}
$$

Each term of the right-hand side of this expression has, as a function of $k$, its support contained in a compact set independent of $s \geqq 0$ and $\varepsilon= \pm$. It follows now from (2.1b), (2.2a), $P_{\varepsilon}(k) \hat{\alpha}_{-\varepsilon}(k)=0$ and Lemma 3.16 that

$$
\left\|F_{3}(s)-\lambda_{3}(s)\right\|_{W^{n, 2}} \leqq C(1+s)^{-2}, \quad s \geqq 0,
$$

for every $n \geqq 0$.

It follows from the above dsicussion and from Theorem 3.17 that the limit of each term in the sum on the right-hand side of

$$
\int_{T}^{t} F_{1}(s) d s=\int_{T}^{t}\left(\left(F_{1}(s)-F_{2}(s)\right)+\left(F_{2}(s)-F_{3}(s)\right)+\left(F_{3}(s)-\lambda_{3}(s)\right)+\lambda_{3}(s)\right) d s
$$

exists in $W^{n, 2}, n \geqq 0$ as $T \rightarrow \infty$. This proves the first statement of the theorem.

To prove the second statement we first introduce

$$
g_{\varepsilon}(k, s)=i\left(\varepsilon \omega(k)-\frac{\partial S_{\varepsilon}(k, s)}{\partial s}\right) P_{\varepsilon}(k)\left(\beta_{-\varepsilon}(s)\right)(k) e^{i\left(S_{-\varepsilon}(k, s)-S_{\varepsilon}(k, s)+2 \varepsilon \omega(k) s\right)} .
$$


It follows as above from (2.1b), (2.2a), and $P_{\varepsilon}(k) \hat{\alpha}_{-\varepsilon}(k)=0$ that $\left|g_{\varepsilon}(k, s)\right| \leqq C(1+s)^{-2}$ for $s \geqq 0$ and $k \in R^{3}$. Using also that $(\partial / \partial s) S_{\varepsilon}(k, s)=L_{\varepsilon}\left(S^{(3)}, \beta^{(3)}, s\right)$ and Lemma 3.15 iii) and then (2.2b), it follows that

$$
\left|\frac{\partial^{2} S_{\varepsilon}(k, s)}{\partial s^{2}}\right| \leqq C(1+s)^{-2} \quad \text { for all } k \in R^{3}, s \geqq 0,
$$

which shows that

$$
\left|\frac{\partial g_{\varepsilon}(k, s)}{\partial s}\right| \leqq C(1+s)^{-3} \quad \text { for all } k \in R^{3}, s \geqq 0 .
$$

Partial integration gives:

$$
\begin{aligned}
& \int_{\infty}^{t} g_{\varepsilon}(k, s) e^{-2 i \varepsilon \omega(k) s} d s=g_{\varepsilon}(k, t)(-2 i \varepsilon \omega(k))^{-1} e^{-2 i \varepsilon \omega(k) t} \\
& +\int_{\infty}^{t}(2 i \varepsilon \omega(k))^{-1} e^{-2 i \varepsilon \omega(k) s} \frac{\partial}{\partial s} g_{\varepsilon}(k, s) d s .
\end{aligned}
$$

By the above estimate for $g_{\varepsilon}(k, s)$ and $(\partial / \partial s) g_{\varepsilon}(k, s)$ we get:

$$
\left|\int_{\infty}^{t} g_{\varepsilon}(k, s) e^{-2 i \varepsilon \omega(k) s} d s\right| \leqq C(1+t)^{-2} .
$$

Equations (3.44), (3.46), and (3.47) give:

$$
\left\|\int_{\infty}^{t}\left(F_{3}(s)-\lambda_{2}(s)-\lambda_{3}(s)\right) d s\right\|_{W^{n, 2}} \leqq C_{n}(1+t)^{-2}
$$

for all $t \geqq 0, n \geqq 0$.

By (3.43), (3.48) and Proposition 3.18 we get:

$$
\left\|\int_{\infty}^{t} W_{0}(t, 0)\left(F_{1}(s)-\lambda_{2}(s)\right) d s-T W_{0}(t, 0) T l(t)\right\|_{W^{n, 2}} \leqq C_{n}(1+t)^{-2}
$$

for all $n \geqq 0, t \geqq 0$.

The definitions (3.40) and (3.34) of $\beta$ give:

$$
\beta_{\varepsilon}(t)-\alpha_{\varepsilon}-P_{\varepsilon}(\partial) \int_{\infty}^{t} \lambda_{2}(S, \beta, s) d s-l_{\varepsilon}(S, \beta, t)=\beta_{\varepsilon}^{(3)}(t)-\beta_{\varepsilon}^{(4)}(t) .
$$

Application of Lemma 3.19 [the estimate (3.37b)] to the last expression gives

$$
\left\|\beta_{\varepsilon}(t)-\alpha_{\varepsilon}-P_{\varepsilon}(\partial) \int_{\infty}^{t} \lambda_{2}(S, \beta, s) d s-l_{\varepsilon}(S, \beta, t)\right\|_{W^{n, 2}} \leqq C_{n}(1+t)^{-3}
$$

for all $n \geqq 0, t \geqq 0$. Finally the inequalities (3.49) and (3.50) give

$$
\begin{aligned}
& \left\|W_{0}(t, 0) \alpha+\int_{\infty}^{t} W_{0}(t, 0) F_{1}(s) d s-\phi(t)\right\|_{W^{n, 2}} \\
& \leqq \\
& \quad\left\|\int_{\infty}^{t} W_{0}(t, 0)\left(F_{1}(s)-\lambda_{2}(s)\right) d s-T W_{0}(t, 0) T l(t)\right\|_{W^{n, 2}} \\
& \quad+\sum_{\varepsilon}\left\|\alpha_{\varepsilon}+P_{\varepsilon}(\partial) \int_{\infty}^{t} \lambda_{2}(s) d s+l_{\varepsilon}(t)-\beta_{\varepsilon}(t)\right\|_{W^{n, 2}} \leqq C_{n}(1+t)^{-2}
\end{aligned}
$$

for all $n \geqq 0$ and $t \geqq 0$. Q.E.D.

We can now sum up the results of this paragraph. 
Theorem 3.23. For $(a, \dot{a}, \alpha) \in E_{+}$, let $S$ and $\beta$ be given by formula (3.40). Then $(B, \phi)$ is an approximative solution of Eq. (1.10), where $\phi$ is given by (2.3) and $B^{v}(t)=\dot{\mu}(t) a^{v}$ $+\mu(t) \dot{a}^{v}+G^{v}(t)$ with $G^{v}$ given by (3.14).

Proof. We have to verify that the conditions of Definition 2.2 are satisfied.

By (2.6) we have

$$
\left\|M^{v}(t)\right\|_{W^{n, 2}} \leqq\left\|\int_{\infty}^{t} d s \mu(t-s) \overline{\phi(s)} \gamma^{v} \phi(s)-G^{v}(t)\right\|_{W^{n, 2}}, \quad t \geqq 0 .
$$

From formulas (3.1) and (3.18) one obtains that $\left\|M^{v}(t)\right\|_{W^{n, 2}} \leqq\left\|H^{v}(t)\right\|_{W^{n, 2}}$. Theorem 3.6 proves that condition i) of Definition 2.2 is satisfied. Similarly one gets

$$
\left\|\frac{d}{d t} M^{v}(t)\right\|_{W^{n, 2}} \leqq\left\|\frac{d}{d t} H^{v}(t)\right\|_{W^{n, 2}}
$$

and once more Theorem 3.6 proves that condition ii) of Definition 2.2 is satisfied. The condition iii) of the definition is satisfied as is seen from Theorem 3.2 ii). Q.E.D.

\section{Appendix A}

The function $t \rightarrow\left\|W_{0}(t, 0) \beta(t)\right\|_{L^{\infty}}, t \geqq 0$ has the same decay as solutions of the free Dirac equation.

Proposition A.1. Let $S$ satisfy (2.1a) and let $\beta$ satisfy (2.2a) and (2.2c). Then

$$
\sup _{t \geqq 0}(1+t)^{3 / 2}\left\|W_{0}(t, 0) \beta(t)\right\|_{L^{\infty}}+\infty \text {. }
$$

Proof. For $\varepsilon=+$ or - introduce

$$
F_{\varepsilon}(x, t)=\int d k e^{i v_{\varepsilon}(x / t, k, t) t} \widehat{\beta}_{\varepsilon}(k, t), \quad x \in R^{3}, t \geqq 0,
$$

where

$$
v_{\varepsilon}(y, k, t)=y \cdot k+t^{-1} S_{\varepsilon}(k, t), \quad \hat{\beta}_{\varepsilon}(k, t)=\left(\beta_{\varepsilon}(t)\right)^{\wedge}(k),
$$

and denote by

$$
D_{\alpha}=\left\{(x, t) \in R^{4}|| x|/| t \mid \leqq \alpha, t \geqq 0\right\},
$$

where $\alpha \geqq 0$. For the compact set $K$ satisfying (2.2c) choose $\alpha_{0}$ such that

$$
\sup _{k \in K}(|k| / \omega(k))<\alpha_{0}<1 \text {. }
$$

Equation $\nabla_{p} v_{\varepsilon}(y, p, t)=0,|y| \leqq \alpha_{0}$ reads explicitly

$$
y+\varepsilon p(\omega(p))^{-1}+t^{-1} \nabla_{p} \varrho_{\varepsilon}(p, t)=0, \quad|y| \leqq \alpha_{0},
$$

where $S_{\varepsilon}(p, t)=\varepsilon \omega(p) t+\varrho_{\varepsilon}(p, t)$.

When $\varrho_{\varepsilon}=0$, there is a unique solution

$$
p_{0}(y, t)=-\varepsilon m y\left(1-|y|^{2}\right)^{-1 / 2} .
$$


The equation for $p$ can be rewritten as $p=p_{0}+f(p)$, where

$$
f(p)=-\varepsilon\left(\omega(p)-\omega\left(p_{0}\right)\right)\left(y+t^{-1} \nabla_{p} \varrho_{\varepsilon}(p, t)\right)-\varepsilon\left(1-|y|^{2}\right)^{-1 / 2} t^{-1} \nabla_{p} \varrho_{\varepsilon}(p, t) .
$$

If $t$ is sufficiently large, then $f$ is a contraction. Indeed as

$$
\begin{aligned}
\left|f\left(p_{1}\right)-f\left(p_{2}\right)\right| \leqq & \left|\omega\left(p_{1}\right)-\omega\left(p_{2}\right)\right||y| \\
& +t^{-1}\left|\omega\left(p_{1}\right) \nabla_{p_{1}} \varrho_{\varepsilon}\left(p_{1}, t\right)-\omega\left(p_{2}\right) \nabla_{p_{2}} \varrho_{\varepsilon}\left(p_{2}, t\right)\right| \\
& +\left(1-|y|^{2}\right)^{-1 / 2} t^{-1}\left|\nabla_{p_{1}} \varrho_{\varepsilon}\left(p_{1}, t\right)-\nabla_{p_{2}} \varrho_{\varepsilon}\left(p_{2}, t\right)\right|
\end{aligned}
$$

and

$$
\left|\omega\left(p_{1}\right)-\omega\left(p_{2}\right)\right||y| \leqq \alpha_{0} \frac{\left|p_{1}+p_{2}\right|}{\omega\left(p_{1}\right)+\omega\left(p_{2}\right)}\left|p_{1}-p_{2}\right| \leqq \alpha_{0}\left|p_{1}-p_{2}\right|
$$

it follows that $\left|f\left(p_{1}\right)-f\left(p_{2}\right)\right| \leqq v\left|p_{1}-p_{2}\right|$ with $v<1$, in a compact neighbourhood of $p_{0}$ if $t$ is sufficiently large.

It follows now from Theorem 7.7 of [9] that

$$
\sup _{(x, t) \in D_{\alpha_{0}}}\left|t^{3 / 2} F_{\varepsilon}(x, t)\right|<+\infty .
$$

If $|y| \geqq \alpha_{0}$ there is then, by the construction of $\alpha_{0}, \delta>0$ such that

$$
\inf _{p \in K}\left(\alpha_{0}-|p|(\omega(p))^{-1}\right) \geqq 2 \delta \text {. }
$$

By choosing $s_{0}$ large enough so that $\sup _{p \in K}\left|\nabla_{p} \varrho_{\varepsilon}(p, t)\right| t^{-1} \leqq \delta$ for $t \geqq s_{0}$, we get

$$
\inf _{p \in K}\left|\nabla_{p} v_{\varepsilon}(y, p, t)\right| \geqq \inf _{p \in K}|y|-|p|(\omega(p))^{-1}-\left|\nabla_{p} \varrho_{\varepsilon}(p, t)\right| \geqq \delta
$$

for all $y$ with $|y| \geqq \alpha_{0}$ and $t \geqq s_{0}$. Hence for these $y$ and $t$, Eq. (A.1) has no solution. Theorem 7.7.1 of [9] together with the fact

$$
\sup _{\substack{p \in K \\ t \geq 0 \\ y \geqq 0}}\left|\nabla_{p}^{n+1} v_{\varepsilon}(y, p, t)\right|<+\infty \quad \text { for } n \geqq 0,
$$

and (A.3) give

$$
\sup _{(x, t) \in R^{4}-D_{\alpha_{0}}}\left|t^{n} F_{\varepsilon}(x, t)\right|<+\infty \text { for } n \geqq 0 .
$$

Inequalities (A.2), (A.4) and $\left|F_{\varepsilon}(x, t)\right| \leqq \int d k\left|\hat{\beta}_{\varepsilon}(k, t)\right| \leqq C$ prove the proposition. Q.E.D.

Important Remark. After the completion of the present article, the authors have noted that the wave operator $\Omega:(a, \dot{a}, \alpha) \rightarrow(A(0), \dot{A}(0), \psi(0))$, introduced at the end the introduction, is $C^{\infty}$ from $E_{+}$[see (1.11)] to $E$ (see Theorem 2.3). This fact follows from the differentiability of $M, \Phi$, and $\mathscr{K}$ in $(a, \dot{a}, \alpha)$, [see (2.6), (2.7), and (2.9)], and from Eq. (2.13). Further regularity properties of $\Omega$, including that of analyticity, will be proved in a forthcoming publication. 


\section{References}

1. Anderson, R.L., Taflin, E.: The Benjamin-Ono equation, recursivity of linearization maps, Lax Pairs. Lett. Math. Phys. 9, 299-311 (1985)

2. Chadam, J.M.: Global solutions of the Cauchy problem for the (classical) coupled MaxwellDirac equations in one space dimension. J. Funct. Anal. 13, 173-184 (1973)

3. Chadam, J.M.: On the Cauchy problem for the coupled Maxwell-Dirac equations. J. Math. Phys. 13, 597-604 (1972)

4. Choquet-Bruhat, Y.: Solutions globales des equations de Maxwell-Dirac-Klein-Gordon (masses nulles). C.R. Acad. Sci. Paris 292, Ser. I, 153-158 (1981)

5. Flato, M., Pinczon, G., Simon, J.: Non-linear representations of Lie groups. Ann. Sci. Ec. Norm. Super. 10, 405-418 (1977)

6. Flato, M., Simon, J.: Non-linear equations and covariance. Lett. Math. Phys. 2, 155-160 (1979)

7. Flato, M., Simon, J.: On a linearization program of non-linear field equations. Phys. Lett. B 94, 518-522 (1980)

8. Gross, L.: The Cauchy problem for the coupled Maxwell and Dirac equations. Commun. Pure Appl. Math. 19, 1-5 (1966)

9. Hörmander, L.: The analysis of linear partial differential operators. Berlin, Heidelberg, New York: Springer 1983

10. Kato, T.: Quasilinear equations of evolution, with application to partial differential equations. Lecture Notes in Mathematics, Vol. 448. Berlin, Heidelberg, New York: Springer 1975, pp. $25-70$

11. Simon, J.C.H., Taflin, E.: Wave operators and analytic solutions for systems of non-linear Klein-Gordon equations and of non-linear Schrödinger equations. Commun. Math. Phys. 99, 541-562 (1985)

12. Taflin, E.: Analytic linearization, Hamiltonian formalism, and infinite sequences of constants of motion for the Burgers equation. Phys. Rev. Lett. 47, 1425-1428 (1981)

13. Taflin, E.: Analytic linearization of the Korteweg-de Vries equation. Pac. J. Math. 108, 203-220 (1983)

Communicated by E. Lieb

Received February 1, 1987 
